\title{
Classifying Descents According to Equivalence mod k
}

\author{
Sergey Kitaev \\ Institute of Mathematics \\ Reykjavík University \\ IS-103 Reykjavík, Iceland \\ sergey@ru.is
}

\author{
Jeffrey Remmel* \\ Department of Mathematics \\ University of California, San Diego \\ La Jolla, CA 92093-0112. USA \\ remmel@math.ucsd.edu
}

Submitted: Apr 20, 2006; Accepted: Jul 17, 2006; Published: Aug 3, 2006

MR Subject Classifications: 05A15, 05E05

\begin{abstract}
In [6] the authors refine the well-known permutation statistic "descent" by fixing parity of (exactly) one of the descent's numbers. In this paper, we generalize the results of [6] by studying descents according to whether the first or the second element in a descent pair is divisible by $k$ for some $k \geq 2$. We provide either an explicit or an inclusion-exclusion type formula for the distribution of the new statistics. Based on our results we obtain combinatorial proofs of a number of remarkable identities. We also provide bijective proofs of some of our results and state a number of open problems.
\end{abstract}

Keywords: permutation statistics, descents, distribution, bijection

\section{Introduction}

The descent set, Des $(\pi)$, of a permutation $\pi=\pi_{1} \pi_{2} \cdots \pi_{n}$ is the set of indices $i$ for which $\pi_{i}>\pi_{i+1}$. The number of descents in a permutation $\pi$, denoted by $\operatorname{des}(\pi)$, is a classical permutation statistic. This statistic was first studied by MacMahon [9] almost a hundred years ago, and it still plays an important role in the study of permutation statistics.

The Eulerian numbers $A(n, k)$ count the number of permutations in the symmetric group $\mathcal{S}_{n}$ with $k$ descents and they are the coefficients of the Eulerian polynomials $A_{n}(t)$

defined by $A_{n}(t)=\sum_{\pi \in \mathcal{S}_{n}} t^{1+\operatorname{des}(\pi)}$. The Eulerian polynomials satisfy the identity

$$
\sum_{k \geq 0} k^{n} t^{k}=\frac{A_{n}(t)}{(1-t)^{n+1}} .
$$

\footnotetext{
*Supported in part by NSF grant DMS 0400507
} 
For more properties of the Eulerian polynomials see [1].

In [6], the authors considered the problem of counting descents according to the parity of the first or second element of the descent pair. That is, let $\mathcal{S}_{n}$ be the set of permutations of $\{1, \ldots, n\}, N=\{0,1,2, \ldots\}$ be the set of natural numbers, $E=\{0,2,4, \ldots$,$\} be the$ set of even numbers, $O=\{1,3,5, \ldots\}$ be the set of odd numbers, and for any statement $A$, let $\chi(A)=1$ if $A$ is true and $\chi(A)=0$ if $A$ is false. Then for any $\sigma \in \mathcal{S}_{n}$, define

- $\overleftarrow{D e s}_{E}(\sigma)=\left\{i: \sigma_{i}>\sigma_{i+1} \& \sigma_{i} \in E\right\}$ and $\overleftarrow{\operatorname{des}}_{E}(\sigma)=\left|\overleftarrow{D e s}_{E}(\sigma)\right|$

- $\overrightarrow{D e s}_{E}(\sigma)=\left\{i: \sigma_{i}>\sigma_{i+1} \& \sigma_{i+1} \in E\right\}$ and $\overrightarrow{d e s}_{E}(\sigma)=\left|\overrightarrow{D e s}_{E}(\sigma)\right|$

- $\overleftarrow{D e s}_{O}(\sigma)=\left\{i: \sigma_{i}>\sigma_{i+1} \& \sigma_{i} \in O\right\}$ and $\overleftarrow{\operatorname{des}}_{O}(\sigma)=\left|\overleftarrow{D e s}_{O}(\sigma)\right|$

- $\overrightarrow{D e s}_{O}(\sigma)=\left\{i: \sigma_{i}>\sigma_{i+1} \& \sigma_{i+1} \in O\right\}$ and $\overrightarrow{d e s}_{O}(\sigma)=\left|\overrightarrow{D e s}_{O}(\sigma)\right|$

Kitaev and Remmel [6] studied the following polynomials:

1. $R_{n}(x)=\sum_{\sigma \in S_{n}} x^{\overleftarrow{\operatorname{des}_{E}}(\sigma)}=\sum_{k=0}^{n} R_{k, n} x^{k}$

2. $P_{n}(x, z)=\sum_{\sigma \in S_{n}} x^{\overrightarrow{\operatorname{des}}_{E}(\sigma)} z^{\chi\left(\sigma_{1} \in E\right)}=\sum_{k=0}^{n} \sum_{j=0}^{1} P_{j, k, n} z^{j} x^{k}$,

3. $M_{n}(x)=\sum_{\sigma \in S_{n}} x^{\overleftarrow{\operatorname{des}}(\sigma)}=\sum_{k=0}^{n} M_{k, n} x^{k}$, and

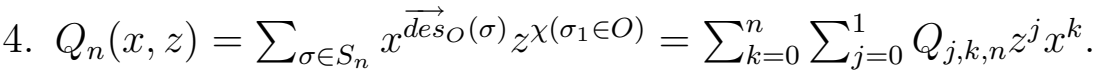

There are some surprisingly simple formulas for the coefficients of these polynomials. For example, the following results are proved in [6].

\section{Theorem 1.}

$$
\begin{aligned}
R_{k, 2 n} & =\left(\begin{array}{l}
n \\
k
\end{array}\right)^{2}(n !)^{2}, \\
R_{k, 2 n+1} & =\frac{1}{k+1}\left(\begin{array}{l}
n \\
k
\end{array}\right)^{2}((n+1) !)^{2}, \\
P_{1, k, 2 n} & =\left(\begin{array}{c}
n-1 \\
k
\end{array}\right)\left(\begin{array}{c}
n \\
k+1
\end{array}\right)(n !)^{2}, \\
P_{0, k, 2 n} & =\left(\begin{array}{c}
n-1 \\
k
\end{array}\right)\left(\begin{array}{l}
n \\
k
\end{array}\right)(n !)^{2}, \\
P_{0, k, 2 n+1} & =(k+1)\left(\begin{array}{l}
n \\
k
\end{array}\right)\left(\begin{array}{l}
n+1 \\
k+1
\end{array}\right)(n !)^{2}=(n+1)\left(\begin{array}{l}
n \\
k
\end{array}\right)^{2}(n !)^{2}, \text { and } \\
P_{0, k, 2 n+1} & =(n+1)\left(\begin{array}{l}
n \\
k
\end{array}\right)^{2}(n !)^{2} .
\end{aligned}
$$


In this paper, we generalize Kitaev and Remmel's results by studying the problem of counting descents according to whether the first or the second element in a descent pair is divisible by $k$ for $k \geq 2$. For any $k>0$, let $k N=\{0, k, 2 k, 3 k, \ldots\}$. Given a set $X \subseteq N=\{0,1, \ldots\}$ and any $\sigma=\sigma_{1} \cdots \sigma_{n} \in \mathcal{S}_{n}$, we define the following:

- $\overleftarrow{D e s}_{X}(\sigma)=\left\{i: \sigma_{i}>\sigma_{i+1} \& \sigma_{i} \in X\right\}$ and $\overleftarrow{\operatorname{des}}_{X}(\sigma)=\left|\overleftarrow{D e s}_{X}(\sigma)\right|$

- $\overrightarrow{D e s}_{X}(\sigma)=\left\{i: \sigma_{i}>\sigma_{i+1} \& \sigma_{i+1} \in X\right\}$ and $\overrightarrow{d e s}_{X}(\sigma)=\left|\overrightarrow{D e s}_{X}(\sigma)\right|$;

- $A_{n}^{(k)}(x)=\sum_{\sigma \in \mathcal{S}_{n}} x^{\overleftarrow{d e s}_{k N}(\sigma)}=\sum_{j=0}^{\left\lfloor\frac{n}{k}\right\rfloor} A_{j, n}^{(k)} x^{j}$

- $B_{n}^{(k)}(x)=\sum_{\sigma \in \mathcal{S}_{n}} x^{\overrightarrow{\operatorname{des}}_{k N}(\sigma)}=\sum_{j=0}^{\left\lfloor\frac{n}{k}\right\rfloor} B_{j, n}^{(k)} x^{j}$.

- $B_{n}^{(k)}(x, z)=\sum_{\sigma \in \mathcal{S}_{n}} x^{\overrightarrow{\mathrm{des}}_{k N}(\sigma)} z^{\chi\left(\sigma_{1} \in k N\right)}=\sum_{j=0}^{\left\lfloor\frac{n}{k}\right\rfloor} \sum_{i=0}^{1} B_{i, j, n}^{(k)} z^{i} x^{j}$.

Remark 1. Note that setting $k=1$ gives us (usual) descents, providing $A_{n}^{(1)}(x)=$ $B_{n}^{(1)}(x)=A_{n}(x)$, whereas setting $k=2$ gives $\overleftarrow{D e s}_{E}(\sigma)$ and $\overrightarrow{D e s}_{E}(\sigma)$ studied in $[6]$.

The goal of this paper is to derive closed formulas for the coefficients of these polynomials. When $k>2$, our formulas are considerably more complicated than the formulas in the $k=2$ case. In fact, in most cases, we can derive two distinct formulas for the coefficients of these polynomials. We shall see that there are simple recursions for the coefficients of the polynomials $A_{k n+j}^{(k)}(x), B_{k n+j}^{(k)}(x)$, and $B_{k n+j}^{(k)}(x, z)$ for $0 \leq j \leq k-1$. In fact, we can derive two different formulas for the coefficients of our polynomials by iterating the recursions starting with the constant term and by iterating the recursions starting with the highest coefficient. For example, we shall prove the following theorem.

Theorem 2. For all $k \geq 2, n \geq 0$, and $0 \leq j \leq k-1$,

$$
\begin{aligned}
& A_{s, k n+j}^{(k)} \\
& =((k-1) n+j) ! \sum_{r=0}^{s}(-1)^{s-r}\left(\begin{array}{c}
(k-1) n+j+r \\
r
\end{array}\right)\left(\begin{array}{c}
k n+j+1 \\
s-r
\end{array}\right) \prod_{i=0}^{n-1}(r+1+j+(k-1) i) \\
& =((k-1) n+j) ! \sum_{r=0}^{n-s}(-1)^{n-s-r}\left(\begin{array}{c}
(k-1) n+j+r \\
r
\end{array}\right)\left(\begin{array}{c}
k n+j+1 \\
n-s-r
\end{array}\right) \prod_{i=1}^{n}(r+(k-1) i) .
\end{aligned}
$$

What is remarkable about these two different formulas for $A_{k n+j}^{(k)}$ is that they lead to a number of identities that are interesting in their own right. For example, it follows from Theorem 2 that for all $k \geq 2, n \geq 0$, and $0 \leq j \leq k-1$,

$$
\begin{aligned}
\sum_{r=0}^{s}(-1)^{s-r} & \left(\begin{array}{c}
(k-1) n+j+r \\
r
\end{array}\right)\left(\begin{array}{c}
k n+j+1 \\
s-r
\end{array}\right) \prod_{i=0}^{n-1}(r+1+j+(k-1) i) \\
= & \sum_{r=0}^{n-s}(-1)^{n-s-r}\left(\begin{array}{c}
(k-1) n+j+r \\
r
\end{array}\right)\left(\begin{array}{c}
k n+j+1 \\
n-s-r
\end{array}\right) \prod_{i=1}^{n}(r+(k-1) i) .
\end{aligned}
$$


Even in the case $k=2$, we get some remarkable identities. For example, it follows from Theorems 1 and 2 that for all $n \geq s$,

$$
\begin{aligned}
\left(\begin{array}{c}
n \\
s
\end{array}\right)^{2}(n !) & =\sum_{r=0}^{s}(-1)^{s-r}\left(\begin{array}{c}
n+r \\
r
\end{array}\right)\left(\begin{array}{c}
2 n+1 \\
s-r
\end{array}\right) \prod_{i=0}^{n-1}(r+1+i) \\
& =\sum_{r=0}^{n-s}(-1)^{n-s-r}\left(\begin{array}{c}
n+r \\
r
\end{array}\right)\left(\begin{array}{c}
2 n+1 \\
n-s-r
\end{array}\right) \prod_{i=1}^{n}(r+i) .
\end{aligned}
$$

It turns out that both of these identities can be derived by using certain hypergeometric series identities. For example, we will show how (2) can be derived from Saalcshütz's identity. Jim Haglund [4] suggested that (1) should follow from Gasper's transformation [2] of hypergeometric series of Karlsson-Minton type. This is indeed the case but we will not include such a derivation in this paper since (1) is a special case of wider class of identities that arise by studying the problem of enumerating permutations according to the number of pattern matches where the equivalence classes of the elements modulo $k$ for $k \geq 2$ are taken into account, see [7]. A general derivation of this wider class of identities from the Gasper's transformation of hypergeometric series of Karlsson-Minton type will appear in a subsequent paper [8].

Given any permutation $\sigma=\sigma_{1} \cdots \sigma_{n} \in \mathcal{S}_{n}$, we label the possible positions of where we can insert $n+1$ to get a permutation in $\mathcal{S}_{n+1}$ from left to right with 0 to $n$, i.e., inserting $n+1$ in position 0 means that we insert $n+1$ at the start of $\sigma$ and for $i \geq 1$, inserting $n+1$ in position $i$ means we insert $n+1$ immediately after $\sigma_{i}$. In such a situation, we let $\sigma^{(i)}$ denote the permutation of $\mathcal{S}_{n+1}$ that results by inserting $n+1$ in position $i$.

Let $\sigma^{c}=\left(n+1-\sigma_{1}\right)\left(n+1-\sigma_{2}\right) \cdots\left(n+1-\sigma_{n}\right)$ denote the complement of $\sigma$. Clearly, if $n$ is odd, then, for all $i, \sigma_{i}$ and $n+1-\sigma_{i}$ have the same parity, whereas they have opposite parity if $n$ is even. However, if $k \geq 3$, then complementation does not preserve equivalences classes mod $k$. The reverse of $\sigma$ is the permutation $\sigma^{r}=\sigma_{n} \sigma_{n-1} \cdots \sigma_{1}$.

The outline of this paper is as follows. In section 2 , we shall give explicit formulas for the coefficients $A_{0, k n+j}^{(k)}$ and $A_{n, k n+j}^{(k)}$ for all $k \geq 2, n \geq 0$, and $j \in\{0, \ldots, k-1\}$. Then we shall develop a set of recursions for the coefficients $A_{s, k n+j}^{(k)}$ and use these recursions to derive our two different formulas for the coefficients of $A_{k n+j}^{(k)}(x)$ for all $n \geq 0$ and $j \in\{0, \ldots, k-1\}$. In section 3 , we shall give explicit formulas for the coefficients $B_{0, k n+j}^{(k)}, B_{0,0, k n+j}^{(k)}, B_{1,0, k n+j}^{(k)}, B_{n, k n+j}^{(k)}, B_{0, n, k n+j}^{(k)}$, and $B_{1, n, k n+j}^{(k)}$ for all $k \geq 2, n \geq 0$, and $j \in\{0, \ldots, k-1\}$. Then we shall develop a set of recursions for the coefficients $B_{i, s, k n+j}^{(k)}$ and use these recursions to derive inclusion-exclusion type formulas for the coefficients of $B_{s, k n+j}^{(k)}, B_{0, s, k n+j}^{(k)}$, and $B_{1, s, k n+j}^{(k)}$ for all $n \geq 0$ and $j \in\{0, \ldots, k-1\}$. Based on such formulas, we shall derive a number of remarkable identities (see Theorem 17). In section 4 , we shall consider some natural bijective questions that arise from our results. Finally, in section 5 , we shall discuss a number of open questions. 


\section{Properties of $A_{n}^{(k)}(x)$}

In this section, we shall study the properties of the polynomials $A_{n}^{(k)}(x)$. For instance, here are some examples of the polynomials $A_{n}^{(3)}(x)$.

$$
\begin{aligned}
& A_{1}^{(3)}(x)=1 \\
& A_{2}^{(3)}(x)=2 . \\
& A_{3}^{(3)}(x)=2+4 x \\
& A_{4}^{(3)}(x)=12+12 x . \\
& A_{5}^{(3)}(x)=72+48 x \\
& A_{6}^{(3)}(x)=72+456 x+192 x^{2} . \\
& A_{7}^{(3)}(x)=960+3120 x+960 x^{2} . \\
& A_{8}^{(3)}(x)=10800+23760 x+5760 x^{2} . \\
& A_{9}^{(3)}(x)=10800+133920 x+183600 x^{2}+34560 x^{3} . \\
& A_{10}^{(3)}(x)=241920+1572480 x+1572480 x^{2}+241920 x^{3} . \\
& A_{11}^{(3)}(x)=4233600+18869760 x+14878080 x^{2}+1935360 x^{3} . \\
& A_{12}^{(3)}(x)=4233600+84309120 x+233331840 x^{2}+141644160 x^{3} 15482880 x^{4} . \\
& A_{13}^{(3)}(x)=139345920+1478373120 x+2991582720 x^{2}+1478373120 x^{3}+139345920 x^{4} . \\
& A_{14}^{(3)}(x)=3429216000+25202016000 x+40334112000 x^{2}+16819488000 x^{3}+1393459200 x^{4} . \\
& A_{15}^{(3)}(x)=3429216000+98413056000 x+448628544000 x^{2}+551287296000 x^{3}+191981664000 x^{4}+ \\
& 1393459200 x^{5} .
\end{aligned}
$$

By Theorem 2, we know that $A_{s, 3 n+j}^{(3)}$ is divisible by $(2 n+j)$ !. However, if we consider $A_{4,15}^{(3)} /(10 !)=191981664000 /(10 !)=52905$, then one can check that the prime factorization of 52905 is $3 \cdot 5 \cdot 3527$. Thus the prime 3527 divides $A_{4,15}^{(3)}$ so that we can not expect that we will get formulas for $A_{s, 3 n+j}^{(3)}$ that are as simple as the formulas that appear in Theorem 1 for the polynomials $A_{s, 2 n+j}^{(2)}$.

For the rest of this paper, we shall assume that $k \geq 2$.

For $j=1, \ldots, k-1$, let $\Delta_{k n+j}$ be the operator which sends $x^{s}$ to $s x^{s-1}+(k n+j-s) x^{s}$ and $\Gamma_{k n+k}$ be the operator that sends $x^{s}$ to $(s+1) x^{s}+(k n+k-1-s) x^{s+1}$. Then we have the following. 
Theorem 3. The polynomials $\left\{A_{n}^{(k)}(x)\right\}_{n \geq 1}$ satisfy the following recursions.

(1) $A_{1}^{(k)}(x)=1$,

(2) For $j=1, \ldots, k-1, A_{k n+j}^{(k)}(x)=\Delta_{k n+j}\left(A_{k n+j-1}^{(k)}(x)\right)$ for $n \geq 0$, and

(3) $A_{k n+k}^{(k)}(x)=\Gamma_{k n+k}\left(A_{k n+k-1}^{(k)}(x)\right)$ for $n \geq 1$.

Proof. Part (1) is trivial.

For part (2), fix $j$ such that $1 \leq j \leq k-1$. Now suppose $\sigma=\sigma_{1} \cdots \sigma_{k n+j-1} \in \mathcal{S}_{k n+j-1}$ and $\overleftarrow{d e s}_{k N}(\sigma)=s$. It is then easy to see that if we insert $k n+j$ in position $i$ where $i \in \overleftarrow{D e s}_{k N}(\sigma)$, then $\overleftarrow{d e s}_{E}\left(\sigma^{(i)}\right)=s-1$. However, if we insert $k n+j$ in position $i$ where $i \notin \overleftarrow{D e s}_{k N}(\sigma)$, then $\overleftarrow{d e s}_{k N}\left(\sigma^{(i)}\right)=s$. Thus $\left\{\sigma^{(i)}: i=0, \ldots, k n+j-1\right\}$ gives a contribution of $s x^{s-1}+(k n+j-s) x^{s}$ to $A_{k n+j}^{(k)}(x)$.

For part (3), suppose $\sigma=\sigma_{1} \cdots \sigma_{k n+k-1} \in \mathcal{S}_{k n+k-1}$ and $\overleftarrow{\mathrm{des}}_{k N}(\sigma)=s$. It is then easy to see that if we insert $k n+k$ in position $i$ where $i \in \overleftarrow{D e s}_{E}(\sigma)$ or $i=k n+k-1$, then $\overleftarrow{d e s}_{E}\left(\sigma^{(i)}\right)=s$. Similarly if we insert $k n+k$ in position $i$ where $i \notin \overleftarrow{D e s}_{k N}(\sigma) \cup\{k n+k-1\}$, then $\overleftarrow{\operatorname{des}}_{k n}\left(\sigma^{(i)}\right)=s+1$. Thus $\left\{\sigma^{(i)}: i=0, \ldots, k n+k-1\right\}$ gives a contribution of $(s+1) x^{s}+(k n+k-(s+1)) x^{s+1}$ to $A_{k n+k}^{(k)}(x)$.

Note that we can rewrite Theorem 1 as saying that

1. $A_{1}^{(k)}(x)=1$,

2. For $j=1, \ldots, k-1, A_{k n+j}^{(k)}(x)=(1-x) \frac{d}{d x}\left(A_{k n+j-1}^{(k)}(x)\right)+(k n+j) A_{k n+j-1}^{(k)}(x)$ for $n \geq 0$, and

3. $A_{k n+k}^{(k)}(x)=\left(x-x^{2}\right) \frac{d}{d x}\left(A_{k n+k-1}^{(k)}(x)\right)+(1+x(k n+k-1)) A_{k n+k-1}^{(k)}(x)$ for $n \geq 1$.

There are simple formulas for the lowest and the highest coefficients in the polynomials $A_{k n+j}^{(k)}$ and we can give direct combinatorial proofs of such formulas.

Theorem 4. We have

(a) $A_{0, k n+j}^{(k)}=((k-1) n+j) ! \prod_{i=0}^{n-1}(j+1+i(k-1))$ for $0 \leq j \leq k-1$;

(b) $A_{n, k n+j}^{(k)}=(n(k-1)+j) !(k-1)^{n} n !$ for $0 \leq j \leq k-1$.

Proof. It is easy to see that both (a) and (b) hold for $n=1$.

To prove (a), fix $j, 0 \leq j \leq k-1$, and suppose that $\sigma=\sigma_{1} \cdots \sigma_{k n+j}$ is such that $\overleftarrow{d e s}_{k N}(\sigma)=0$. Then we can factor any such permutation into blocks by reading the permutation from left to right and cutting after each number which is not divisible by $k$. For example if $j=0, k=3$, and $\sigma=111245367891012$, then the blocks of $\sigma$ would be $11,1,2,4,5,367,8,910,12$. 
There may be a block of numbers which are divisible by $k$ at the end which are arranged in increasing order. We call this final block the $\infty$-th block. Every other block must end with a number $s k+i$ where $0 \leq s \leq n-1$ and $1 \leq i \leq k-1$ and can be preceded by any subset of numbers which are divisible by $k$ and which are less than $s k+i$ arranged in increasing order. We call such a block the $(s k+i)$-th block. It is then easy to see that there are $\prod_{i=0}^{n-1}(j+1+i(k-1))$ ways to put the numbers $k, 2 k, \ldots, n k$ into the blocks. That is, $k n$ must go in either the blocks $k n+1, \ldots, k n+j$ or in the $\infty$-th block so that there are $1+j$ choices for the block in which to place $k n$. Then $k(n-1)$ can either go in the blocks $k(n-1)+1, k(n-1)+2, \ldots, k(n-1)+k-1, k n+1, \ldots, k n+j$ or the $\infty$-block so that there are $j+1+(k-1)$ choices for the block that contains $k(n-1)$. More generally, $k(n-i)$ can go in any blocks $k(n-i)+1, \ldots, k(n-i)+k-1, k(n-i+$ $1)+1, \ldots, k(n-i+1)+k-1, \ldots, k(n-1)+1, \ldots, k(n-1)+k-1, k n+1, \ldots, k n+j$ or the $\infty$-block so that are $j+1+i(k-1)$ choices for the block that contains $k(n-i)$. Once we have arranged the numbers which are divisible by $k$ into blocks, it is easy to see that we can arrange blocks $s k+i$ where $0 \leq s \leq n-1$ and $1 \leq i \leq k-1$ plus the blocks $k n+1, \ldots, k n+j$ in any order and still get a permutation $\sigma$ with $\overleftarrow{d e s}_{k N}(\sigma)=0$. It thus follows that there are $((k-1) n+j) ! \prod_{i=0}^{n-1}(j+1+i(k-1))$ such permutations.

To prove (b) fix $j, 0 \leq j \leq k-1$, and suppose that $\sigma=\sigma_{1} \cdots \sigma_{k n+j}$ is such that $\overleftarrow{\mathrm{des}}_{k N}(\sigma)=n$. Then, as above, we can factor any such permutation into blocks by reading the permutation from left to right and cutting after each number which is not divisible by $k$. One can see that, unlike the case where $\overleftarrow{d e s}_{k N}(\sigma)=0$, there can be no numbers which are divisible by $k$ at the end since that would force at least one number which is divisible by $k$ to not start a descent. Thus the $\infty$-th block must be empty. Similarly, it is easy to see that the blocks $k n+1, \ldots, k n+j$ must be singletons. Next if $0 \leq s \leq n-1$ and $1 \leq j \leq k-1$ and there are numbers which are divisible by $k$ in the $(s k+j)$-th block, i.e. the block that ends with $s k+j$, then those numbers must all be greater than $s k+j$ and they must be arranged in decreasing order.

It is then easy to see that there are $(k-1)^{n}(n !)$ ways to put the numbers $k, 2 k, \ldots, n k$ into blocks. That is, $k n$ may go in any of the blocks $s k+j$ where $0 \leq s \leq n-1$ and $1 \leq j \leq k-1$ so that there are $(k-1) n$ choices for the block that contains $k n$. Then $k(n-1)$ can go in any of the blocks $s k+j$ where $0 \leq s \leq n-2$ and $1 \leq j \leq k-1$ so that there are $(k-1)(n-1)$ choices for the block that contains $k(n-1)$, etc. After we have partitioned the numbers which are divisible by $k$ into their respective blocks, we must arrange the numbers which are divisible by $k$ in each block in decreasing order so that there are a total $(k-1)^{n} n$ ! ways to partition the numbers which are divisible by $k$ into the blocks. Once we have arranged the numbers which are divisible by $k$ into blocks, it is easy to see that we can arrange blocks $s k+j$ where $0 \leq s \leq n-1$ and $1 \leq j \leq k-1$ plus the blocks $k n+1, \ldots, k n+j$ in any order an still get a permutation $\sigma$ with $\overleftarrow{\operatorname{des}}_{k N}(\sigma)=n$. It thus follows that there are $(n(k-1)+j) !(k-1)^{n} n$ ! such permutations.

It is easy to see from Theorem 3 that we have two following recursions for the coefficients $A_{s, n}^{(k)}$. 
For $1 \leq j \leq k-1$

$$
A_{s, k n+j}^{(k)}=(k n+j-s) A_{s, k n+j-1}^{(k)}+(s+1) A_{s+1, k n+j-1}^{(k)}
$$

and

$$
A_{s, k n+k}^{(k)}=(1+s) A_{s, k n+k-1}^{(k)}+(k n+k-s) A_{s-1, k n+k-1}^{(k)} .
$$

The following theorem provides an inclusion-exclusion type formula for $A_{s, k n+j}^{(k)}$ which can be obtained by iterating the recursions (3) and (4) starting with our formulas for $A_{0, k n+j}^{(k)}$.

Theorem 5. For all $0 \leq j \leq k-1$ and all $n \geq 0$, we have

$$
\begin{aligned}
& A_{s, k n+j}^{(k)}= \\
& ((k-1) n+j) !\left[\sum_{r=0}^{s}(-1)^{s-r}\left(\begin{array}{c}
(k-1) n+j+r \\
r
\end{array}\right)\left(\begin{array}{c}
k n+j+1 \\
s-r
\end{array}\right) \prod_{i=0}^{n-1}(r+1+j+(k-1) i)\right] .
\end{aligned}
$$

Proof. We shall prove this formula by induction on $s$. Note that Theorem 4 shows that our formula for $A_{s, k n+j}^{(k)}$ holds when $s=0$ for all $n \geq 0$ and $0 \leq j \leq k-1$.

Now assume by induction that our formula for $A_{s, k n+j}^{(k)}$ is true for all $n \geq 0$ and $0 \leq j \leq k-1$. Then we shall prove that it holds for $A_{s+1, k n+j}^{(k)}$ for all $n \geq 0$ and $0 \leq j \leq k-1$. Note that by recursion (3), we have for $1 \leq j \leq k-1$,

$$
(s+1) A_{s+1, k n+j-1}^{(k)}=A_{s, k n+j}^{(k)}-(k n+j-s) A_{s, k n+j-1}^{(k)} .
$$

Thus

$$
\begin{aligned}
(s+1) & A_{s+1, k n+j-1}^{(k)} \\
= & ((k-1) n+j) !\left[\sum_{r=0}^{s}(-1)^{s-r}\left(\begin{array}{c}
(k-1) n+j+r \\
r
\end{array}\right)\left(\begin{array}{c}
k n+j+1 \\
s-r
\end{array}\right) \prod_{i=0}^{n-1}(r+1+j+(k-1) i)\right] \\
& -(k n+j-s)((k-1) n+j-1) ! \times \\
& {\left[\sum_{r=0}^{s}(-1)^{s-r}\left(\begin{array}{c}
(k-1) n+j-1+r \\
r
\end{array}\right)\left(\begin{array}{c}
k n+j \\
s-r
\end{array}\right) \prod_{i=0}^{n-1}(r+j+(k-1) i)\right] . }
\end{aligned}
$$

It follows that

$$
\begin{aligned}
& \frac{A_{s+1, k n+j-1}^{(k)}}{((k-1) n+j-1) !} \\
& =\frac{((k-1) n+j)}{s+1}\left[\sum_{r=0}^{s}(-1)^{s-r}\left(\begin{array}{c}
(k-1) n+j+r \\
r
\end{array}\right)\left(\begin{array}{c}
k n+j+1 \\
s-r
\end{array}\right) \prod_{i=0}^{n-1}(r+1+j+(k-1) i)\right] \\
& \quad-\frac{(k n+j-s)}{s+1}\left[\sum_{r=0}^{s}(-1)^{s-r}\left(\begin{array}{c}
(k-1) n+j-1+r \\
r
\end{array}\right)\left(\begin{array}{c}
k n+j \\
s-r
\end{array}\right) \prod_{i=0}^{n-1}(r+j+(k-1) i)\right] .
\end{aligned}
$$


We can divide the terms on the RHS of (5) into the three parts. The $r=s$ term from the first summand on the RHS of (5) gives

$$
\begin{aligned}
& \frac{((k-1) n+j)}{s+1}\left(\begin{array}{c}
(k-1) n+j+s \\
s
\end{array}\right) \prod_{i=0}^{n-1}(s+1+j+(k-1) i) \\
& =\left(\begin{array}{c}
(k-1) n+j+s \\
s+1
\end{array}\right) \prod_{i=0}^{n-1}(s+1+j+(k-1) i) \\
& =\left(\begin{array}{c}
(k-1) n+(j-1)+s+1 \\
s+1
\end{array}\right) \prod_{i=0}^{n-1}((s+1)+1+(j-1)+(k-1) i) .
\end{aligned}
$$

The $r=0$ term from the second summand on the RHS of (5) gives

$$
\begin{aligned}
(-1)^{s+1} \frac{(k n+j-s)}{s+1}\left(\begin{array}{c}
k n+j \\
s
\end{array}\right) \prod_{i=0}^{n-1}(j+(k-1) i) \\
=(-1)^{s+1}\left(\begin{array}{c}
k n+j \\
s+1
\end{array}\right) \prod_{i=0}^{n-1}(j+(k-1) i) \\
=(-1)^{s+1}\left(\begin{array}{c}
k n+(j-1)+1 \\
s+1
\end{array}\right) \prod_{i=0}^{n-1}(1+(j-1)+(k-1) i) .
\end{aligned}
$$

Finally we can organize the remaining terms of each summand according to the factor $\prod_{i=0}^{n-1}(r+j+(k-1) i)$ to get

$$
\begin{aligned}
\sum_{r=1}^{s}(-1)^{s+1-r} \prod_{i=0}^{n-1}(r+j+(k-1) i) \times\left[\frac{k n+j-s}{s+1}\left(\begin{array}{c}
(k-1) n+j-1+r \\
r
\end{array}\right)\left(\begin{array}{c}
k n+j \\
s-r
\end{array}\right)\right. & \left.+\frac{(k-1) n+j}{s+1}\left(\begin{array}{c}
(k-1) n+j+r-1 \\
r-1
\end{array}\right)\left(\begin{array}{c}
k n+j+1 \\
s-(r-1)
\end{array}\right)\right] \\
= & \sum_{r=1}^{s}(-1)^{s+1-r} \frac{((k-1) n+j+r-1) \downarrow_{r}}{(r-1) !} \frac{(k n+j) \downarrow_{s-r}}{(s-r) !} \frac{1}{s+1} \prod_{i=0}^{n-1}(r+j+(k-1) i) \times \\
= & \left.\quad \frac{k n+j-s}{r}+\frac{k n+j+1}{s+1-r}\right] \\
& \sum_{r=1}^{s}(-1)^{s+1-r} \frac{((k-1) n+j+r-1) \downarrow_{r}}{(r-1) !} \frac{(k n+j) \downarrow_{s-r}}{(s-r) !} \frac{1}{s+1} \prod_{i=0}^{n-1}(r+j+(k-1) i) \times \\
& \frac{(k n+j-s+r)}{r(s+1-r)}
\end{aligned}
$$




$$
\begin{aligned}
& =\sum_{r=1}^{s}(-1)^{s+1-r}\left(\begin{array}{c}
(k-1) n+j+r-1 \\
r
\end{array}\right)\left(\begin{array}{c}
k n+j \\
s+1-r
\end{array}\right) \prod_{i=0}^{n-1}(r+j+(k-1) i) \\
& =\sum_{r=1}^{s}(-1)^{s+1-r}\left(\begin{array}{c}
(k-1) n+j-1+r \\
r
\end{array}\right)\left(\begin{array}{c}
k n+(j-1)+1 \\
s+1-r
\end{array}\right) \prod_{i=0}^{n-1}(r+1+(j-1)+(k-1) i),
\end{aligned}
$$

where $x \downarrow_{r}=x(x-1) \cdots(x-r+1)$. Thus if we combine (6), (7), and (8), we get

$$
\begin{aligned}
& \frac{A_{s+1, k n+j-1}^{(k)}}{((k-1) n+j-1) !}= \\
& \sum_{r=0}^{s+1}(-1)^{s+1-r}\left(\begin{array}{c}
(k-1) n+(j-1)+r \\
r
\end{array}\right)\left(\begin{array}{c}
k n+(j-1)+1 \\
s+1-r
\end{array}\right) \prod_{i=0}^{n-1}(r+1+(j-1)+(k-1) i)
\end{aligned}
$$

as desired. Thus we have proved our formula for $A_{s+1, k n+j}^{(k)}$ holds for all $n$ and for all $0 \leq j \leq k-2$.

Next we verify that our formula holds for $A_{s+1, k n+k-1}^{(k)}$. By recursion (4), we have

$$
(1+t) A_{t, k n+k-1}^{(k)}=A_{t, k n+k}^{(k)}-(k n+k-t) A_{t-1, k n+k-1}^{(k)} .
$$

Putting $t=s+1$ in (9), we have that

$$
(s+2) A_{s+1, k n+k-1}^{(k)}=A_{s+1, k n+k}^{(k)}-(k n+k-(s+1)) A_{s, k n+k-1}^{(k)} .
$$

Thus using the formula for $A_{s+1, k n+k}^{(k)}$ that we just proved and our induction hypothesis, we have

$$
\begin{aligned}
& (s+2) A_{s+1, k n+k-1}^{(k)}=((k-1)(n+1)) ! \times \\
& \quad\left[\sum_{r=0}^{s+1}(-1)^{s+1-r}\left(\begin{array}{c}
(k-1)(n+1)+r \\
r
\end{array}\right)\left(\begin{array}{c}
k(n+1)+1 \\
s+1-r
\end{array}\right) \prod_{i=0}^{n}(r+1+(k-1) i)\right] \\
& \quad-(k n+k-(s+1))((k-1) n+(k-1)) ! \times \\
& \quad\left[\sum_{r=0}^{s}(-1)^{s-r}\left(\begin{array}{c}
(k-1) n+k-1+r \\
r
\end{array}\right)\left(\begin{array}{c}
k n+k-1+1 \\
s-r
\end{array}\right) \prod_{i=0}^{n-1}(r+1+(k-1)+(k-1) i)\right] .
\end{aligned}
$$

Thus

$$
\begin{aligned}
& \frac{A_{s+1, k n+k-1}^{(k)}}{(k-1) n+k-1) !}= \\
& \frac{1}{s+2}\left[\sum_{r=0}^{s+1}(-1)^{s+1-r}\left(\begin{array}{c}
(k-1)(n+1)+r \\
r
\end{array}\right)\left(\begin{array}{c}
k(n+1)+1 \\
s+1-r
\end{array}\right) \prod_{i=0}^{n}(r+1+(k-1) i)\right]
\end{aligned}
$$




$$
\begin{aligned}
& -\frac{(k n+k-(s+1))}{s+2} \times \\
& {\left[\sum_{r=0}^{s}(-1)^{s-r}\left(\begin{array}{c}
(k-1) n+k-1+r \\
r
\end{array}\right)\left(\begin{array}{c}
k n+k \\
s-r
\end{array}\right) \prod_{i=0}^{n-1}(r+1+(k-1)+(k-1) i)\right] .}
\end{aligned}
$$

Note that $\prod_{i=0}^{n}(r+1+(k-1) i)=(r+1) \prod_{i=0}^{n-1}(r+1+(k-1)+(k-1) i)$. Thus we can divide the RHS of (11) into two terms. The first term comes from the $r=s+1$ in the first summand on the RHS of (11) and yields

$$
\begin{gathered}
\frac{1}{s+2}\left(\begin{array}{c}
(k-1)(n+1)+s+1 \\
s+1
\end{array}\right)(s+2) \prod_{i=0}^{n-1}(s+1+1+(k-1)+(k-1) i) \\
=\left(\begin{array}{c}
(k-1)(n+1)+s+1 \\
s+1
\end{array}\right) \prod_{i=0}^{n-1}(s+1+1+(k-1)+(k-1) i) .
\end{gathered}
$$

The remaining terms can be organized according to the factor $\prod_{i=0}^{n-1}(r+1+(k-1)+$ $(k-1) i)$ to give

$$
\begin{gathered}
\sum_{r=0}^{s}(-1)^{s+1-r}\left(\begin{array}{c}
(k-1) n+(k-1)+r \\
r
\end{array}\right) \prod_{i=0}^{n-1}(r+1+(k-1)+(k-1) i) \frac{1}{s+2} \times \\
{\left[(1+r)\left(\begin{array}{c}
k n+k+1 \\
s+1-r
\end{array}\right)+(k n+k-(s+1))\left(\begin{array}{c}
k n+k \\
s-r
\end{array}\right)\right] .}
\end{gathered}
$$

But

$$
\begin{aligned}
{[(1+r)} & \left.\left(\begin{array}{c}
k n+k+1 \\
s+1-r
\end{array}\right)+(k n+k-(s+1))\left(\begin{array}{c}
k n+k \\
s-r
\end{array}\right)\right] \\
& =\frac{(k n+k) \downarrow_{s-r}}{(s+1-r) !}[(1+r)(k n+k+1)+(s+1-r)(k n+k-(s+1))] \\
& =\frac{(k n+k) \downarrow_{s-r}}{(s+1-r) !}[(s+2)(k n+k)+(r+1)-(s+1-r)(s+1)] \\
& =\frac{(k n+k) \downarrow_{s-r}}{(s+1-r) !}[(s+2)(k n+k)-(s+2)(s-r)] \\
& =(s+2) \frac{(k n+k) \downarrow_{s+1-r}}{(s+1-r) !} \\
= & (s+2)\left(\begin{array}{c}
k n+k \\
s+1-r
\end{array}\right) .
\end{aligned}
$$


Substituting (14) in (13), we get that (13) is equivalent to

$$
\sum_{r=0}^{s}(-1)^{s+1-r}\left(\begin{array}{c}
(k-1) n+(k-1)+r \\
r
\end{array}\right)\left(\begin{array}{c}
k n+k \\
s+1-r
\end{array}\right) \prod_{i=0}^{n-1}(r+1+(k-1)+(k-1) i) .
$$

Finally, combining (12) and (15), we obtain

$$
\begin{aligned}
& \frac{A_{s+1, k n+k-1}^{(k)}}{((k-1) n+k-1) !}= \\
& \sum_{r=0}^{s+1}(-1)^{s+1-r}\left(\begin{array}{c}
(k-1) n+(k-1)+r \\
r
\end{array}\right)\left(\begin{array}{c}
k n+(k-1)+1 \\
s+1-r
\end{array}\right) \prod_{i=0}^{n-1}(r+1+(k-1)+(k-1) i)
\end{aligned}
$$

as desired.

As a corollary to Theorem 5 we get combinatorial proofs for two special cases of the Saalschütz's identity, which in terms of generalized hypergeometric functions can be written as

$$
{ }_{3} F_{2}\left[\begin{array}{ccc}
a & b & c \\
d & e & ; 1
\end{array}\right]=\frac{(d-a)_{|c|}(d-b)_{|c|}}{d_{|c|}(d-a-b)_{|c|}}
$$

where $d+e=a+b+c+1$ and $c$ is a negative integer ${ }^{1}$ (see [10] pages 43 and 126).

Corollary 1. The following identities hold:

$$
\begin{gathered}
\left(\begin{array}{l}
n \\
s
\end{array}\right)^{2}=\sum_{r=0}^{s}(-1)^{s-r}\left(\begin{array}{c}
n+r \\
r
\end{array}\right)^{2}\left(\begin{array}{c}
2 n+1 \\
s-r
\end{array}\right) \\
\left(\begin{array}{l}
n \\
s
\end{array}\right)\left(\begin{array}{c}
n+1 \\
s+1
\end{array}\right)=\sum_{r=0}^{s}(-1)^{s-r}\left(\begin{array}{c}
n+r+1 \\
r
\end{array}\right)\left(\begin{array}{c}
n+r+1 \\
r+1
\end{array}\right)\left(\begin{array}{c}
2 n+2 \\
s-r
\end{array}\right) .
\end{gathered}
$$

Proof. The RHS of the first identity is $A_{s, 2 n}^{(2)} /(n !)^{2}$ (we use Theorem 5 for $k=2$ and $j=0)$. However, as stated in Theorem 1 in the introduction, we proved $A_{s, 2 n}^{(2)}=R_{s, 2 n}=$ $(n !)^{2}\left(\begin{array}{l}n \\ s\end{array}\right)^{2}$ in [6]. The RHS of the second identity is $A_{s, 2 n+1}^{(2)} /(n !(n+1) !)$ and by Theorem 1 , $A_{s, 2 n+1}^{(2)}=R_{s, 2 n+1}=n !(n+1) !\left(\begin{array}{l}n \\ s\end{array}\right)\left(\begin{array}{c}n+1 \\ s+1\end{array}\right)$.

Remarkably, there is a second inclusion-exclusion type formula for the coefficients $A_{s, k n+j}^{(k)}$ which can be obtained by iterating the recursions (3) and (4) starting with our formulas for $A_{n, k n+j}^{(k)}$.

\footnotetext{
${ }^{1}$ For the first identity in Corollary $1, a=n+1, b=n+1, c=-s, d=1$, and $e=2 n+2-s$; for the second identity there, $a=n+2, b=n+2, c=-s, d=2$, and $e=2 n+3-s$.
} 
Theorem 6. For all $0 \leq j \leq k-1$ and $0 \leq s \leq n$,

$$
\begin{aligned}
& A_{n-s, k n+j}^{(k)}= \\
& ((k-1) n+j) !\left[\sum_{r=0}^{s}(-1)^{s-r}\left(\begin{array}{c}
(k-1) n+j+r \\
r
\end{array}\right)\left(\begin{array}{c}
k n+j+1 \\
s-r
\end{array}\right) \prod_{i=1}^{n}(r+(k-1) i)\right] .
\end{aligned}
$$

Proof. Again we proceed by induction on $s$. By Theorem 4, we have proved our formula for $A_{n-s, k n+j}^{(k)}$ in the case where $s=0$ for all $n \geq 0$ and $0 \leq j \leq k-1$.

Now assume that $s>0$ and that the theorem hold for all $s^{\prime}<s$ by induction. Note that for $n=0$ and $j=0, \ldots, k-1$, our formula asserts that

$$
\begin{aligned}
A_{-s, j}^{(k)} & =j ! \sum_{r=0}^{s}(-1)^{s-r}\left(\begin{array}{c}
j+r \\
r
\end{array}\right)\left(\begin{array}{c}
j+1 \\
s-r
\end{array}\right) \\
& =\left.j !\left(\sum_{n \geq 0}\left(\begin{array}{c}
j+n \\
n
\end{array}\right) x^{n}\right)\left(\sum_{m \geq 0}(-1)^{m}\left(\begin{array}{c}
j+1 \\
m
\end{array}\right) x^{m}\right)\right|_{x^{s}} \\
& =\left.j ! \frac{1}{(1-x)^{j+1}}(1-x)^{j+1}\right|_{x^{s}}=0,
\end{aligned}
$$

where $\left.\right|_{x^{s}}$ denotes the coefficient to $x^{s}$, so that our formula holds for $n=0$ for $j=$ $0, \ldots, k-1$.

Next, by induction, assume that the our formula holds for $s$ for $n^{\prime}<n$ and $j=$ $0, \ldots, k-1$. Recall by $(4)$

$$
A_{s, k n+k}^{(k)}=(1+s) A_{s, k n+k-1}^{(k)}+(k n+k-s) A_{s-1, k n+k-1}^{(k)} .
$$

Replacing $n$ by $n-1$ and $s$ by $n-s$ in (17), we obtain that

$$
A_{n-s, k n}^{(k)}=(1+n-s) A_{n-1-(s-1), k(n-1)+k-1}^{(k)}+((k-1) n+s) A_{n-1-s, k(n-1)+k-1}^{(k)} .
$$

Thus by induction, we obtain that

$$
\begin{aligned}
& A_{n-s, k n}^{(k)}=(1+n-s)((k-1) n) ! \times \\
& \quad \sum_{r=0}^{s-1}(-1)^{s-1-r}\left(\begin{array}{c}
(k-1)(n-1)+k-1+r \\
r
\end{array}\right)\left(\begin{array}{c}
k(n-1)+k-1+1 \\
s-1-r
\end{array}\right) \prod_{i=1}^{n-1}(r+(k-1) i) \\
& \quad-((k-1) n+s)((k-1) n) ! \times \\
& \quad \sum_{r=0}^{s}(-1)^{s-r}\left(\begin{array}{c}
(k-1)(n-1)+k-1+r \\
r
\end{array}\right)\left(\begin{array}{c}
k(n-1)+k-1+1 \\
s-r
\end{array}\right) \prod_{i=1}^{n-1}(r+(k-1) i) .
\end{aligned}
$$

Hence 


$$
\begin{aligned}
& \frac{A_{n-s, k n}^{(k)}}{((k-1) n) !} \\
& \quad(1+n-s) \sum_{r=0}^{s-1}(-1)^{s-1-r}\left(\begin{array}{c}
(k-1) n+r \\
r
\end{array}\right)\left(\begin{array}{c}
k n \\
s-1-r
\end{array}\right) \prod_{i=1}^{n-1}(r+(k-1) i) \\
& \quad+((k-1) n+s) \sum_{r=0}^{s}(-1)^{s-r}\left(\begin{array}{c}
(k-1) n+r \\
r
\end{array}\right)\left(\begin{array}{c}
k n \\
s-r
\end{array}\right) \prod_{i=1}^{n-1}(r+(k-1) i) .
\end{aligned}
$$

Now we can divide the LHS of (18) into two parts. First $r=s$ term in the second summand is

$$
((k-1) n+s)\left(\begin{array}{c}
(k-1) n+s \\
s
\end{array}\right) \prod_{i=1}^{n-1}(s+(k-1) i)=\left(\begin{array}{c}
(k-1) n+s \\
s
\end{array}\right) \prod_{i=1}^{n}(s+(k-1) i) .
$$

Then we can combine the remaining terms on the LHS of (18) to obtain

$$
\begin{aligned}
& \sum_{r=0}^{s-1}(-1)^{s-r}\left(\begin{array}{c}
(k-1) n+r \\
r
\end{array}\right) \prod_{i=1}^{n}(r+(k-1) i) \times \\
& \frac{1}{r+(k-1) n}\left[((k-1) n+s)\left(\begin{array}{c}
k n \\
s-r
\end{array}\right)-(1+n-s)\left(\begin{array}{c}
k n \\
s-1-r
\end{array}\right)\right] .
\end{aligned}
$$

The term in square brackets in (20) is equal to

$$
\begin{aligned}
& {\left[((k-1) n+s) \frac{(k n) \downarrow_{s-r}}{(s-r) !} \frac{k n+1}{k n+1}-(1+n-s) \frac{(k n) \downarrow_{s-1-r}}{(s-1-r) !} \frac{k n+1}{k n+1} \frac{s-r}{s-r}\right]} \\
& \quad=\frac{1}{k n+1} \frac{(k n+1) \downarrow_{s-r}}{(s-r) !}[((k-1) n+s)(k n-(s-r)+1)-(1+n-s)(s-r)] \\
& \quad=\frac{1}{k n+1}\left(\begin{array}{c}
k n+1 \\
s-r
\end{array}\right)[(k n+1)(r+(k-1) n)] \\
& \quad=(r+(k-1) n)\left(\begin{array}{c}
k n+1 \\
s-r
\end{array}\right) .
\end{aligned}
$$

Thus plugging in (21) into (20), we see that (20) is equal to

$$
\sum_{r=0}^{s-1}(-1)^{s-r}\left(\begin{array}{c}
(k-1) n+r \\
r
\end{array}\right)\left(\begin{array}{c}
k n+1 \\
s-r
\end{array}\right) \prod_{i=1}^{n}(r+(k-1) i) .
$$

Thus we can combine (19) into (22) to obtain that

$$
\frac{A_{n-s, k n}^{(k)}}{((k-1) n) !}=\sum_{r=0}^{s}(-1)^{s-r}\left(\begin{array}{c}
(k-1) n+r \\
r
\end{array}\right)\left(\begin{array}{c}
k n+1 \\
s-r
\end{array}\right) \prod_{i=1}^{n}(r+(k-1) i) .
$$


as desired.

By (3), we have that

$$
A_{s, k n+j}^{(k)}=(k n+j-s) A_{s, k n+j-1}^{(k)}+(s+1) A_{s+1, k n+j-1}^{(k)}
$$

for $1 \leq j \leq k-1$. Replacing $s$ by $n-s$ in (24), we obtain that

$$
A_{n-s, k n+j}^{(k)}=((k-1) n+j+s) A_{n-s, k n+j-1}^{(k)}+(n-s+1) A_{n-(s-1), k n+j-1}^{(k)} .
$$

We can assume by induction that our formula holds for $A_{n-s, k n+j-1}^{(k)}$ so that

$$
\begin{aligned}
& A_{n-s, k n+j}^{(k)}=((k-1) n+j+s)((k-1) n+j-1) ! \times \\
& \quad \sum_{r=0}^{s}(-1)^{s-r}\left(\begin{array}{c}
(k-1) n+j-1+r \\
r
\end{array}\right)\left(\begin{array}{c}
k n+j-1+1 \\
s-r
\end{array}\right) \prod_{i=1}^{n}(r+(k-1) i) \\
& \quad+(n-s+1)((k-1) n+j-1) ! \times \\
& \quad \sum_{r=0}^{s-1}(-1)^{s-1-r}\left(\begin{array}{c}
(k-1) n+j-1+r \\
r
\end{array}\right)\left(\begin{array}{c}
k n+j-1+1 \\
s-1-r
\end{array}\right) \prod_{i=1}^{n}(r+(k-1) i) .
\end{aligned}
$$

Thus

$$
\begin{aligned}
& \frac{A_{n-s, k n+j}^{(k)}}{((k-1) n+j-1) !} \\
= & ((k-1) n+j+s) \sum_{r=0}^{s}(-1)^{s-r}\left(\begin{array}{c}
(k-1) n+j-1+r \\
r
\end{array}\right)\left(\begin{array}{c}
k n+j \\
s-r
\end{array}\right) \prod_{i=1}^{n}(r+(k-1) i) \\
& +(n-s+1) \sum_{r=0}^{s-1}(-1)^{s-1-r}\left(\begin{array}{c}
(k-1) n+j-1+r \\
r
\end{array}\right)\left(\begin{array}{c}
k n+j \\
s-1-r
\end{array}\right) \prod_{i=1}^{n}(r+(k-1) i) .
\end{aligned}
$$

Again, we can divide the LHS of (25) into two terms. The first term, coming from the $r=s$ of the first summand, is

$$
\begin{aligned}
((k-1) n+j+s) & \left(\begin{array}{c}
(k-1) n+j-1+s \\
s
\end{array}\right) \prod_{i=1}^{n}(s+(k-1) i) \\
= & ((k-1) n+j)\left(\begin{array}{c}
(k-1) n+j+s \\
s
\end{array}\right) \prod_{i=1}^{n}(s+(k-1) i)
\end{aligned}
$$

Next, the remaining terms on the LHS of (25) can be combined into

$$
\begin{aligned}
& \sum_{r=0}^{s-1}(-1)^{s-r} \prod_{i=1}^{n}(s+(k-1) i) \times \\
& {\left[((k-1) n+j+s)\left(\begin{array}{c}
(k-1) n+j-1+r \\
r
\end{array}\right)\left(\begin{array}{c}
k n+j \\
s-r
\end{array}\right)-\right.} \\
& \left.(n-s+1)\left(\begin{array}{c}
(k-1) n+j-1+r \\
r
\end{array}\right)\left(\begin{array}{c}
k n+j \\
s-1-r
\end{array}\right)\right] .
\end{aligned}
$$


Now the term in the square brackets in (27) is equal to

$$
\begin{aligned}
&((k-1) n+j+s) \frac{((k-1) n+j+r-1) \downarrow_{r}}{r !} \frac{(k-1) n+j+r}{(k-1) n+j+r} \frac{(k n+j) \downarrow_{s-r}}{(s-r) !} \frac{k n+j+1}{k n+j+1} \\
&-(n-s+1) \frac{((k-1) n+j+r-1) \downarrow_{r}}{(k-1) n+j+r} \frac{(k n+j) \downarrow_{s-1-r}}{(k n+j) n+j+r} \frac{k n+1}{k n+j+1} \frac{s-r}{s-r} \\
&=\frac{((k-1) n+j+r) \downarrow_{r}}{r !} \frac{(k n+j+1) \downarrow_{s-r}}{(s-r) !} \times \\
& {\left[((k-1) n+j+s) \frac{(k-1) n+j}{(k-1) n+j+r} \frac{k n+j-(s-r)+1}{k n+j+1}\right.} \\
&\left.-(n-s+1) \frac{(k-1) n+j}{(k-1) n+j+r} \frac{(s-r)}{k n+j+1}\right] \\
&=\left(\begin{array}{c}
(k-1) n+j+r \\
r
\end{array}\right)\left(\begin{array}{c}
k n+j+1 \\
s-r
\end{array}\right) \frac{(k-1) n+j}{((k-1) n+j+r)(k n+j+1)} \times \\
&=\left(\left(\begin{array}{c}
(k-1) n+j+s)(k n+j-(s-r)+1)-(n-s+1)(s-r)] \\
(k-1) n+j
\end{array}\right.\right. \\
& {[(k-1) n+j+r)(k n+j+1)] } \\
&=((k-1) n+j)\left(\begin{array}{c}
(k-1) n+j+r \\
r
\end{array}\right)\left(\begin{array}{c}
k n+j+1 \\
s-r
\end{array}\right) .
\end{aligned}
$$

Here the fact that $((k-1) n+j+s)(k n+j-(s-r)+1)-(n-s+1)(s-r)=$ $(k-1) n+j+r)(k n+j+1)$ can easily be verified in your favorite computer algebra system. Thus substituting in the result of (28) into (27), we see that (27) is equal to

$$
((k-1) n+j) \sum_{r=0}^{s-1}(-1)^{s-r}\left(\begin{array}{c}
(k-1) n+j+r \\
r
\end{array}\right)\left(\begin{array}{c}
k n+j+1 \\
s-r
\end{array}\right) \prod_{i=1}^{n}(r+(k-1) i) .
$$

Combining (26) and (29), we see that

$$
\begin{aligned}
& \frac{A_{n-s, k n+j}^{(k)}}{((k-1) n+j-1) !}= \\
& ((k-1) n+j) \sum_{r=0}^{s}(-1)^{s-r}\left(\begin{array}{c}
(k-1) n+j+r \\
r
\end{array}\right)\left(\begin{array}{c}
k n+j+1 \\
s-r
\end{array}\right) \prod_{i=1}^{n}(r+(k-1) i)
\end{aligned}
$$

or, equivalently, that

$$
\begin{aligned}
& A_{n-s, k n+j}^{(k)}= \\
& ((k-1) n+j) ! \sum_{r=0}^{s}(-1)^{s-r}\left(\begin{array}{c}
(k-1) n+j+r \\
r
\end{array}\right)\left(\begin{array}{c}
k n+j+1 \\
s-r
\end{array}\right) \prod_{i=1}^{n}(r+(k-1) i)
\end{aligned}
$$

as desired. This completes our induction and hence we have established our formulas for $A_{n-s, k n+j}^{(k)}$ for all $0 \leq j \leq k-1$ and $n \geq 0$. 
Note that if we compare the formulas for $A_{n-s, k n+j}^{(k)}$ from Theorem 5 and from Theorem 6 , we obtain the following identities

Corollary 2. For all $0 \leq j \leq k-1$ and $0 \leq s \leq n$,

$$
\begin{aligned}
& \sum_{r=0}^{s}(-1)^{s-r}\left(\begin{array}{c}
(k-1) n+j+r \\
r
\end{array}\right)\left(\begin{array}{c}
k n+j+1 \\
s-r
\end{array}\right) \prod_{i=1}^{n}(r+(k-1) i) \\
& \quad=\sum_{r=0}^{n-s}(-1)^{n-s-r}\left(\begin{array}{c}
(k-1) n+j+r \\
r
\end{array}\right)\left(\begin{array}{c}
k n+j+1 \\
n-s-r
\end{array}\right) \prod_{i=0}^{n-1}(r+1+j+(k-1) i) .
\end{aligned}
$$

For example, the case $s=0$ of Corollary 2, gives the following identities. For all $n$ and for all $0 \leq j \leq k-1$,

$$
(k-1)^{n}(n !)=\sum_{r=0}^{n}(-1)^{n-r}\left(\begin{array}{c}
(k-1) n+j+r \\
r
\end{array}\right)\left(\begin{array}{c}
k n+j+1 \\
n-r
\end{array}\right) \prod_{i=0}^{n-1}(r+1+j+(k-1) i) .
$$

Similarly, the $s=1$ of Corollary 2, gives the following identities. For all $n$ and for all $0 \leq j \leq k-1$

$$
\begin{aligned}
& ((k-1) n+j+1) \prod_{i=0}^{n}(1+(k-1) i)-(k n+j+1)(k-1)^{n}(n !) \\
& \quad=\sum_{r=0}^{n-1}(-1)^{n-1-r}\left(\begin{array}{c}
(k-1) n+j+r \\
r
\end{array}\right)\left(\begin{array}{c}
k n+j+1 \\
n-1-r
\end{array}\right) \prod_{i=0}^{n-1}(r+n+(k-1) i) .
\end{aligned}
$$

\section{Properties of $B_{n}^{(k)}(x, z)$}

For $0 \leq j \leq k-2$, let $\Theta_{k n+j}$ be the operator that sends $z^{0} x^{s}$ to $(1+s+(k-1) n+j) z^{0} x^{s}+$ $(n-s) z^{0} x^{s+1}$ and $z^{1} x^{s}$ to $(1+s+(k-1) n+j) z^{1} x^{s}+(n-s-1) z^{1} x^{s+1}+z^{0} x^{s+1}$. Also let $\Psi_{k n+k-1}$ be the operator that sends $z^{0} x^{s}$ to $(s+(k-1)(n+1)) z^{0} x^{s}+z^{1} x^{s}+(n-s) z^{0} x^{s+1}$ and $z^{1} x^{s}$ to $(1+s+(k-1)(n+1)) z^{1} x^{s}+(n-s) z^{1} x^{s+1}$. Then we have the following.

Theorem 7. For any $k \geq 2$ and $n \geq 0$,

1. $B_{1}^{(k)}(x, z)=1$,

2. $B_{k n+j+1}^{(k)}(x, z)=\Theta_{k n+j}\left(B_{k n+j}^{(k)}(x, z)\right)$ for $0 \leq j \leq k-2$, and

3. $B_{k n+k}^{(k)}(x, z)=\Psi_{k n+k-1}\left(B_{k n+k-1}^{(k)}(x, z)\right)$. 
Proof. Part (1) is easy to see.

For part (2), suppose we are given a permutation $\sigma=\sigma_{1} \cdots \sigma_{k n+j}$ where $\overrightarrow{d e s}_{k N}(\sigma)=s$ and $\sigma_{1} \notin k N$ so that such a $\sigma$ gives rise to a factor of $z^{0} x^{s}$ in $B_{k n+j}^{(k)}(x, z)$. Then for each $i$ such that $\sigma_{i}>\sigma_{i+1}$ and $\sigma_{i+1} \in k N$, inserting $k n+j+1$ in position $i$ will result in a permutation $\sigma^{(i)}$ with $\overrightarrow{d e s}_{k N}\left(\sigma^{(i)}\right)=s$ and $\sigma_{1}^{(i)} \notin k N$. Similarly if $i=k n+j$ or $i<k n+j$ and $\sigma_{i+1} \notin k N$, then $\overrightarrow{d e s}_{k N}\left(\sigma^{(i)}\right)=s$ and $\sigma_{1}^{(i)} \notin k N$. Finally if $i \notin \overrightarrow{D e s}_{k N}(\sigma)$ and $\sigma_{i+1} \in k N$, then $\overrightarrow{d e s}_{k N}\left(\sigma^{(i)}\right)=s+1$ and $\sigma_{1}^{(i)} \notin k N$. Thus the collection of $\left\{\sigma^{(i)}\right.$ : $i=0, \ldots, k n+j\}$ contributes a factor of $(1+s+(k-1) n+j) z^{0} x^{s}+(n-s) z^{0} x^{s+1}$ to $B_{k n+j+1}^{(k)}(x, z)$.

Next suppose we are given a permutation $\sigma=\sigma_{1} \cdots \sigma_{k n+j}$ such that $\overrightarrow{d e s}_{k N}(\sigma)=s$ and $\sigma_{1} \in k N$ so that such a $\sigma$ gives rise to a factor of $z^{1} x^{s}$ in $B_{k n+j}^{(k)}(x, z)$. Then for each $i$ where $\sigma_{i}>\sigma_{i+1}$ and $\sigma_{i+1} \in k N$, inserting $k n+j+1$ in position $i$ will result in a permutation $\sigma^{(i)}$ with $\overrightarrow{d e s}_{k N}\left(\sigma^{(i)}\right)=s$ and $\sigma_{1}^{(i)} \in k N$. Similarly if $i=k n+j$ or $i<k n+j$ and $\sigma_{i+1} \notin k N$, then $\overrightarrow{d e s}_{k N}\left(\sigma^{(i)}\right)=s$ and $\sigma_{1}^{(i)} \in k N$. If $i>0, i \notin \overrightarrow{D e s}_{k N}(\sigma)$, and $\sigma_{i+1} \in k N$, then $\overrightarrow{d e s}_{k N}\left(\sigma^{(i)}\right)=s+1$ and $\sigma_{1}^{(i)} \in k N$. Finally if $i=0$, then, since $\sigma_{1} \in k N$, $\overrightarrow{d e s}_{k N}\left(\sigma^{(0)}\right)=s+1$ and $\sigma_{1}^{(0)} \notin k N$. Thus the collection of $\left\{\sigma^{(i)}: i=0, \ldots, k n+j\right\}$ contributes a factor of $(1+s+(k-1) n+j) z^{1} x^{s}+(n-s-1) z^{1} x^{s+1}+z^{0} x^{s+1}$ to $B_{k n+j+1}^{(k)}(x, z)$ in this case.

For part (3), suppose $\sigma=\sigma_{1} \cdots \sigma_{k n+k-1}$ is a permutation such that $\overrightarrow{d e s}_{k N}(\sigma)=s$ and $\sigma_{1} \notin k N$ so that such a $\sigma$ gives rise to a factor of $z^{0} x^{s}$ in $B_{k n+k-1}^{(k)}(x, z)$. Then for each $i$ where $\sigma_{i}>\sigma_{i+1}$ and $\sigma_{i+1} \in k N$, inserting $k n+k$ in position $i$ will result in a permutation $\sigma^{(i)}$ such that $\overrightarrow{d e s}_{k N}\left(\sigma^{(i)}\right)=s$ and $\sigma_{1}^{(i)} \notin k N$. Similarly if $i=k n+k-1$ or $i>0$ and $\sigma_{i+1} \notin k N$, then $\overrightarrow{d e s}_{k N}\left(\sigma^{(i)}\right)=s$ and $\sigma_{1}^{(i)} \notin k N$. Since $\sigma_{1} \notin k N$, inserting $k n+k$ in position 0 will result in a permutation $\sigma$ with $\overrightarrow{d e s}_{k N}\left(\sigma^{(0)}\right)=s$ and $\sigma_{1}^{(i)} \in k N$. Finally if $i \notin \overrightarrow{D e s}_{k N}(\sigma)$ and $\sigma_{i+1} \in k N$, then $\overrightarrow{d e s}_{k N}\left(\sigma^{(i)}\right)=s+1$ and $\sigma_{1}^{(i)} \notin k N$. Thus the collection of $\left\{\sigma^{(i)}: i=0, \ldots, k n+k-1\right\}$ contributes a factor of $(s+(k-1) n+k-1) z^{0} x^{s}+z^{1} x^{s}+$ $(n-s) z^{0} x^{s+1}$ to $B_{k n+k}^{(k)}(x, z)$.

Next suppose $\sigma=\sigma_{1} \cdots \sigma_{k n+k-1}$ is a permutation with $\overrightarrow{d e s}_{k N}(\sigma)=s$ and $\sigma_{1} \in k N$ so that such a $\sigma$ gives rise to a factor of $z^{1} x^{s}$ in $B_{k n+k-1}^{(k)}(x, z)$. Then for each $i$ where $\sigma_{i}>\sigma_{i+1}$ and $\sigma_{i+1} \in k N$, inserting $k n+k$ in position $i$ will result in a permutation $\sigma^{(i)}$ such that $\overrightarrow{d e s}_{k N}\left(\sigma^{(i)}\right)=s$ and $\sigma_{1}^{(i)} \in k N$. Similarly if $i=k n+k-1$ or $i<k n+j \sigma_{i+1} \notin k N$, then $\overrightarrow{d e s}_{k N}\left(\sigma^{(i)}\right)=s$ and $\sigma_{1}^{(i)} \in k N$. Finally if $\sigma_{i+1} \in k N$, then $\overrightarrow{d e s}_{k N}\left(\sigma^{(i)}\right)=s+1$ and $\sigma_{1}^{(i)} \in k N$. Thus the collection of $\left\{\sigma^{(i)}: i=0, \ldots, k n+k-1\right\}$ contributes a factor of $(s+(k-1) n+k) z^{1} x^{s}+(n-s) z^{1} x^{s+1}$ to $B_{k n+k}^{(k)}(x, z)$ in this case.

One can also express the actions $\Theta_{k n+j}$ and $\Psi_{k n+k-1}$ in terms of partial differential operators. That is, it is easy to verify that we have the following.

Theorem 8. For any $k \geq 2$ and $n \geq 0$,

$$
\text { 1. } B_{1}^{(k)}(x, z)=1 \text {, }
$$


2. $B_{k n+j+1}^{(k)}(x, z)=\left(x(1-x) \frac{\partial}{\partial x}+x(1-z) \frac{\partial}{\partial z}+n x+(1+(k-1) n+j)\right)\left(B_{k n+j}^{(k)}(x, z)\right)$ for $0 \leq j \leq k-2$, and

3. $B_{k n+k}^{(k)}(x, z)=\left(x(1-x) \frac{\partial}{\partial x}+z(1-z) \frac{\partial}{\partial z}+n x+z+(k-1)(n+1)\right)\left(B_{k n+k-1}^{(k)}(x, z)\right)$.

One can use Theorem 8 to generate $B_{n}^{(k)}(x, z)$ for small $k$ and $n$. Here are some initial values for $B_{n}^{(3)}(x, z)$.

$$
\begin{aligned}
& B_{1}^{(3)}(x, z)=1 . \\
& B_{2}^{(3)}(x, z)=2 . \\
& B_{3}^{(3)}(x, z)=4+2 z . \\
& B_{4}^{(3)}(x, z)=12+6 z+6 x . \\
& B_{5}^{(3)}(x, z)=48+24 z+48 x . \\
& B_{6}^{(3)}(x, z)=192+168 z+288 x+72 x z . \\
& B_{7}^{(3)}(x, z)=960+840 z+2280 x+600 x z+360 x^{2} . \\
& B_{8}^{(3)}(x, z)=5760+5040 z+18720 x+5040 z+5760 x^{2} . \\
& B_{9}^{(3)}(x, z)=34560+41040 z+142560 x+69120 x z+64800 x^{2}+10800 x^{2} z . \\
& B_{10}^{(3)}(x, z)=241920+287280 z+1285200 x+635040 x z+937440 x^{2}+166320 x^{2} z+75600 x^{3} . \\
& B_{11}^{(3)}(x, z)=1935360+2298240 z+12579840 x+6289920 x z+12579840 x^{2}+2298240 x^{2} z+ \\
& \quad 1935360 x^{3} . \\
& B_{12}^{(3)}(x, z)=15482880+22619520 z+119024640 x+82373760 x z+150958080 x^{2}+50440320 x^{2} z+ \\
& 33868800 x^{3}+4233600 x^{3} z .
\end{aligned}
$$

Recall that for all $n, B_{n}^{(k)}(x, z)=\sum_{\sigma \in \mathcal{S}_{n}} x^{\overrightarrow{\operatorname{des}}_{k N}(\sigma)} z^{\chi\left(\sigma_{1} \in k N\right)}=\sum_{j=0}^{\left\lfloor\frac{n}{k}\right\rfloor} \sum_{i=0}^{1} B_{i, j, n}^{(k)} z^{i} x^{j}$ and $B_{s, k n+j}^{(k)}=B_{0, s, k n+j}^{(k)}+B_{1, s, k n+j}^{(k)}$, for all $n$ and $j$ where $0 \leq j \leq k-1$. It is easy to see that Theorem 7 implies to the following recursions.

For $0 \leq j \leq k-2$ and all $n \geq 0$,

$$
\begin{gathered}
B_{0, s, k n+j+1}^{(k)}=(1+s+(k-1) n+j) B_{0, s, k n+j}^{(k)}+(n-s+1) B_{0, s-1, k n+j}^{(k)}+B_{1, s-1, k n+j}^{(k)} \\
B_{1, s, k n+j+1}^{(k)}=(1+s+(k-1) n+j) B_{1, s, k n+j}^{(k)}+(n-s) B_{1, s-1, k n+j}^{(k)}
\end{gathered}
$$

and

$$
B_{s, k n+j+1}^{(k)}=(1+s+(k-1) n+j) B_{s, k n+j}^{(k)}+(n-s+1) B_{s-1, k n+j}^{(k)} .
$$


Similarly, for all $n \geq 0$,

$$
\begin{gathered}
B_{0, s, k n+k}^{(k)}=(s+(k-1)(n+1)) B_{0, s, k n+k-1}^{(k)}+(n-s+1) B_{0, s-1, k n+k-1}^{(k)}, \\
B_{1, s, k n+k}^{(k)}=(1+s+(k-1)(n+1)) B_{1, s, k n+k-1}^{(k)}+(n-s+1) B_{1, s-1, k n+k-1}^{(k)}+B_{0, s, k n+k-1}^{(k)},
\end{gathered}
$$

and

$$
B_{s, k n+k}^{(k)}=(1+s+(k-1)(n+1)) B_{s, k n+k-1}^{(k)}+(n-s+1) B_{s-1, k n+k-1}^{(k)} .
$$

Note that (36) can be obtained from (33) by plugging in $j=k-1$. Next we shall consider some special values of $B_{i, j, n}^{(k)}$ and $B_{j, n}^{(k)}$.

Theorem 9. For all $n \geq 0, k \geq 2$, and $0 \leq j \leq k-1$,

$$
\begin{aligned}
& \text { 1. } B_{0, k n+j}^{(k)}=((k-1) n+j) ! \prod_{i=1}^{n}(1+(k-1) i) \text {, } \\
& \text { 2. } B_{0,0, k n+j}^{(k)}=((k-1) n+j) ! \prod_{i=1}^{n}((k-1) i)=((k-1) n+j) !(k-1)^{n}(n !) \text {, and } \\
& \text { 3. } B_{1,0, k n+j}^{(k)}=((k-1) n+j) !\left(\left(\prod_{i=1}^{n}(1+(k-1) i)\right)-(k-1)^{n}(n !)\right) \text {. }
\end{aligned}
$$

Proof. It is easy to see that (1), (2) and (3) hold for $n=1$.

To prove (1), fix $j$ such that $0 \leq j \leq k-1$ and suppose that $\sigma=\sigma_{1} \cdots \sigma_{k n+j}$ is such that $\overrightarrow{d e s}_{k N}(\sigma)=0$. Then we can factor any such permutation into blocks by reading the permutation from right to left and cutting before each number which is not divisible by $k$. For example if $j=0, k=3$, and $\sigma=111362457891012$, then the blocks of $\sigma$ would be 11, $136,2,4,5,7,89,1012$.

There may be a block of numbers which are divisible by $k$ at the start which are arranged in increasing order. We call this initial block the 0-th block. Every other block must start with a number $s k+i$ where $0 \leq s \leq n$ and $1 \leq i \leq k-1$ and can be followed by any subset of numbers which are divisible by $k$ and which are greater than $s k+i$ arranged in increasing order. We call such a block the $(s k+i)$-th block. It is then easy to see that there are $\prod_{i=1}^{n}(1+i(k-1))$ ways to put the numbers $k, 2 k, \ldots, n k$ into the blocks. That is, $k$ must be placed in one of the blocks $0,1, \ldots, k-1$ so that there are $1+(k-1)$ choices for the block to place $k$. Then $2 k$ must be placed in one of the blocks $0,1, \ldots, k-1, k+1, \ldots, k+k-1$ so that there are $1+2(k-1)$ choices for the block that contains $2 k$. More generally, $i k$ must be placed in one of the blocks $0,1, \ldots, k-1, k+1, \ldots, k+k-1, \ldots,(i-1) k+1, \ldots,(i-1) k+k-1$ so that there are $1+i(k-1)$ choices for the block that contains $i k$. Once we have arranged the numbers which are divisible by $k$ into blocks, it is easy to see that we can arrange blocks $s k+i$ where $0 \leq s \leq n-1$ and $1 \leq i \leq k-1$ plus the blocks $k n+1, \ldots, k n+j$ in any order and still get a permutation $\sigma$ with $\overrightarrow{d e s}_{k N}(\sigma)=0$. It thus follows that there are $((k-1) n+j) ! \prod_{i=1}^{n}(1+i(k-1))$ such permutations so that $B_{0, k n+j}^{(k)}=((k-1) n+j) ! \prod_{i=1}^{n}(1+i(k-1))$.

To prove our formula for $B_{0,0, k n+j}^{(k)}$, we can essentially use the same argument except that in this case we cannot put any elements in the 0 -th block since we want to produce 
permutations that do not start with an element which is divisible by $k$. Thus there are only $\prod_{i=1}^{n}(k-1) i=(k-1)^{n} n$ ! ways to put the elements which are divisible by $k$ into the blocks in this case. Once again, after we have arranged the numbers which are divisible by $k$ into blocks, it is easy to see that we can arrange blocks $s k+i$ where $0 \leq s \leq n-1$ and $1 \leq i \leq k-1$ plus the blocks $k n+1, \ldots, k n+j$ in any order and still get a permutation $\sigma$ with $\overrightarrow{d e s}_{k N}(\sigma)=0$ and $\sigma_{1} \notin k N$. It thus follows that there are $((k-1) n+j) ! \prod_{i=1}^{n}(i(k-1))$ such permutations so that $B_{0,0, k n+j}^{(k)}=((k-1) n+j) ! \prod_{i=1}^{n}(i(k-1))$.

Clearly, part (3) immediately follows from parts (1) and (2).

Theorem 10. We have $B_{n, k n}^{(k)}=B_{0, n, k n}^{(k)}=0$, and for all $n \geq 0, k \geq 2$, and $1 \leq j \leq k-1$,

1. $B_{n, k n+j}^{(k)}=((k-1) n+j) ! \prod_{i=0}^{n-1}(j+(k-1) i)$,

2. $B_{0, n, k n+j}^{(k)}=((k-1) n+j) ! \prod_{i=0}^{n-1}(j+(k-1) i)$, and

3. $B_{1, n, k n+j}^{(k)}=0$.

Proof. It is easy to see that if a permutation $\sigma$ starts with an element which is divisible by $k$, then it cannot be the case that $\overrightarrow{d e s}_{k N}(\sigma)=n$. Thus $B_{1, n, k n+j}^{(k)}=0$ and $B_{n, k n+j}^{(k)}=$ $B_{0, n, k n+j}^{(k)}$. Moreover, $B_{0, n, k n}^{(k)}=0$ since for permutations $\sigma \in \mathcal{S}_{k n}, k n$ cannot be the second element of a descent because it is the largest element in the permutation. So we need only prove (1).

To prove (1), fix $j$ such that $1 \leq j \leq k-1$ and suppose that $\sigma=\sigma_{1} \cdots \sigma_{k n+j}$ is such that $\overrightarrow{d e s}_{k N}(\sigma)=n$. Then, as above, we can factor any such permutation into blocks by reading the permutation from right to left and cutting before each number which is not divisible by $k$. One can see that, unlike the case where $\overrightarrow{d e s}_{k N}(\sigma)=0$, there can be no numbers which are divisible by $k$ at the start so that the 0 -th block must be empty. Next if $0 \leq s \leq n-1$ and $1 \leq i \leq k-1$ and there are numbers which are divisible by $k$ in the $(s k+i)$-th block, then those numbers must all be less than $s k+i$ and they must be arranged in decreasing order. Finally, if there are numbers in any of the blocks $k n+1, \ldots, k n+j$ contains numbers which are divisible by $k$, then those numbers must be arranged in decreasing order. It is then easy to see that there are $\prod_{i=0}^{n-1}(j+(k-1) i)$ ways to put the numbers $k, 2 k, \ldots, n k$ into blocks. That is, $k n$ can only be placed in one of the blocks $k n+1, \ldots, k n+j$ so that there are only $j$ possible choices for the blocks containing $k n$. Then $k(n-1)$ can only be placed in one of the blocks $k(n-1)+1, \ldots, k(n-$ $1)+k-1, k n+1, \ldots, k n+j$ so that there are only $j+(k-1)$ possible choices for the blocks containing $k(n-1)$. In general, $k(n-i)$ can only be placed in one of the blocks $k(n-i)+1, \ldots, k(n-i)+k-1, \ldots, k(n-1)+1, \ldots, k(n-1)+k-1, k n+1, \ldots, k n+j$ so that there are only $j+i(k-1)$ possible choices for the blocks containing $k(n-i)$. Once we have arranged the numbers which are divisible by $k$ into blocks, it is easy to see that we can arrange blocks $s k+i$ where $0 \leq s \leq n-1$ and $1 \leq i \leq k-1$ plus the blocks $k n+1, \ldots, k n+j$ in any order an still get a permutation $\sigma$ with $\overrightarrow{d e s}_{k N}(\sigma)=n$. It thus follows that there are $(n(k-1)+j) ! \prod_{i=0}^{n-1} j+(k-1) i$ such permutations. 
Theorem 11. For all $n \geq 0, k \geq 2$, and $0 \leq j \leq k-1$,

$$
B_{1, n-1, k n+j}^{(k)}=((k-1) n+j) ! \sum_{p=0}^{n-1}\left(\prod_{i=0}^{p-1}(j+(k-1) i)\right)\left(\prod_{i=p+1}^{n-1}(1+j+(k-1) i)\right) .
$$

Proof. Fix $j, 0 \leq j \leq k-1$. Suppose that $\sigma=\sigma_{1} \cdots \sigma_{k n+j}$ is such that $\overrightarrow{d e s}_{k N}(\sigma)=n-1$. Since we are computing $B_{1, n-1, k n+j}^{(k)}$, we need only consider permutations $\sigma$ such that $\sigma_{1} \in k N$. Now if $\overrightarrow{d e s}_{k N}(\sigma)=n-1$, then $\sigma_{1}$ is the only element which is divisible by $k$ and which is not the second element of a descent pair. Thus we will classify our permutations by the first element. That is, suppose $\sigma_{1}=(n-p) k$ for some $0 \leq p \leq n-1$. Then, as above, we can factor any such permutation into blocks by reading the permutation from right to left and cutting before each number which is not divisible by $k$. Thus the 0 -th block must start with $(n-p) k$ which could be followed by elements which are divisible by $k$ and which are less than $(n-p) k$ arranged in decreasing order. Next if $0 \leq s \leq n$ and $1 \leq i \leq k-1$ and there are numbers which are divisible by $k$ in the $(s k+i)$-th block, then those numbers must all be less than $s k+i$ and they must be arranged in decreasing order. Finally, if there are numbers in any of the blocks $k n+1, \ldots, k n+j$ which are divisible by $k$, then those numbers must be arranged in decreasing order. We claim that there are $\left(\prod_{i=0}^{p-1}(j+(k-1) i)\right)\left(\prod_{i=p+1}^{n-1}(1+j+(k-1) i)\right)$ ways to put the numbers $k, 2 k, \ldots,(n-p-1) k,(n-p+1) k, \ldots, n k$ into blocks. That is, suppose that $p \geq 1$ and consider the possibilities for the placements of $k n, k(n-1), \ldots, k(n-p+1)$ into blocks. Clearly, $k n$ can only be placed in one of the blocks $k n+1, \ldots, k n+j$ so that there are only $j$ possible choices for the blocks containing $k n$. Then $k(n-1)$ can only be placed in one of the blocks $k(n-1)+1, \ldots, k(n-1)+k-1, k n+1, \ldots, k n+j$ so that there are only $j+(k-1)$ possible choices for the blocks containing $k(n-1)$. In general, $k(n-i)$ can only be placed in one of the blocks $k(n-i)+1, \ldots, k(n-i)+$ $k-1, \ldots, k(n-1)+1, \ldots, k(n-1)+k-1, k n+1, \ldots, k n+j$ so that there are only $j+i(k-1)$ possible choices for the blocks containing $k(n-i)$. Thus the total number of choices for the placements of $k n, k(n-1), \ldots, k(n-p+1)$ into blocks is $\prod_{i=0}^{p-1}(j+(k-1) i)$. Next consider the possibilities for the placements of $k(n-p-1), k(n-p-2), \ldots, k$ into blocks. For $i \geq 1, k(n-p-i)$ can be placed in the 0 -th block or any of the blocks $k(n-p-i)+1, \ldots, k(n-p-i)+k-1, \ldots, k(n-1)+1, \ldots, k(n-1)+k-1, k n+1, \ldots, k n+j$ so that there are $1+j+(p+i)(k-1)$ possible choices for the blocks containing $k(n-p-i)$. Thus the total number of choices for the placements of $k(n-p-1), k(n-p-2), \ldots, k$ into blocks is $\prod_{i=p+1}^{n-1}(1+j+(k-1) i)$. A similar argument will show that if $\sigma_{1}=k n$, then there $\prod_{i=1}^{n-1}(1+j+(k-1) i)$ ways to place the elements which are divisible by $k$ into the blocks. After we have placed the numbers which are divisible by $k$ into their respective blocks, we must arrange the numbers which are divisible by $k$ in each block in decreasing order so that there are a total $\left(\prod_{i=0}^{p-1}(j+(k-1) i)\right)\left(\prod_{i=p+1}^{n-1}(1+j+(k-1) i)\right)$ ways to place the numbers which are divisible by $k$ into the blocks for a permutation $\sigma$ such that $\sigma_{1}=k(n-p)$ and $\overrightarrow{d e s}_{k N}(\sigma)=n-1$ for $p=0, \ldots, n-1$. Once we have placed the 
numbers which are divisible by $k$ into blocks, it is easy to see that we can arrange blocks $s k+i$ where $0 \leq s \leq n-1$ and $1 \leq i \leq k-1$ plus the blocks $k n+1, \ldots, k n+j$ in any order and still get a permutation $\sigma$ with $\overrightarrow{d e s}_{k N}(\sigma)=n-1$.

It follows that there are $(n(k-1)+j) !\left(\prod_{i=0}^{p-1}(j+(k-1) i)\right)\left(\prod_{i=p+1}^{n-1}(1+j+(k-1) i)\right)$ such permutations starting with $k(n-p)$, and we get the desired result.

We let $\Omega(k, n, j)=\sum_{p=0}^{n-1}\left(\prod_{i=0}^{p-1}(j+(k-1) i)\right)\left(\prod_{i=p+1}^{n-1}(1+j+(k-1) i)\right)$. It turns out that there is another expression for $\Omega(k, n, j)$ which will be useful for our later developments.

Lemma 12. For all $n \geq 1, k \geq 2$, and $r \geq 0$,

$$
\begin{aligned}
\Omega(k, n, r) & =\sum_{p=0}^{n-1}\left(\prod_{i=0}^{p-1}(r+(k-1) i)\right)\left(\prod_{i=p+1}^{n-1}(1+r+(k-1) i)\right) \\
& =\left(\prod_{i=0}^{n-1}(1+r+(k-1) i)\right)-\left(\prod_{i=0}^{n-1}(r+(k-1) i)\right) .
\end{aligned}
$$

Proof. Note that for $n=1, \sum_{p=0}^{1-1}\left(\prod_{i=0}^{p-1}(r+(k-1) i)\right)\left(\prod_{i=p+1}^{1-1}(1+r+(k-1) i)\right)=1$ since both products involved in the sum are empty. However, $\left(\prod_{i=0}^{1-1}(1+r+(k-1) i)\right)-$ $\left(\prod_{i=0}^{1-1}(r+(k-1) i)\right)=(1+r)-r=1$ so that our formula holds for all $r$ when $n=1$.

Next suppose that $m>1$ and that our formula holds for all $r$ when $n=m-1$. Then

$$
\begin{aligned}
\left(\prod_{i=0}^{m-1}(1+r+(k-1) i)\right)-\left(\prod_{i=0}^{m-1}(r+(k-1) i)\right) \\
=(1+r)\left(\prod_{i=1}^{m-1}(1+r+(k-1) i)\right)-r\left(\prod_{i=1}^{m-1}(r+(k-1) i)\right) \\
=\left(\prod_{i=1}^{m-1}(1+r+(k-1) i)\right) \\
\quad+r\left(\left(\prod_{i=0}^{m-2}(1+r+(k-1)+(k-1) i)\right)-\left(\prod_{i=0}^{m-2}(r+(k-1)+(k-1) i)\right)\right) \\
=\quad\left(\prod_{i=1}^{m-1}(1+r+(k-1) i)\right) \\
\quad+r \sum_{p=0}^{m-2}\left(\prod_{i=0}^{p-1}(r+(k-1)+(k-1) i)\right)\left(\prod_{i=p+1}^{m-2}(1+r+(k-1)+(k-1) i)\right)
\end{aligned}
$$




$$
\begin{aligned}
= & \left(\prod_{i=1}^{m-1}(1+r+(k-1) i)\right) \\
& +r \sum_{p=0}^{m-2}\left(\prod_{i=1}^{p}(r+(k-1) i)\right)\left(\prod_{i=p+2}^{m-1}(1+r+(k-1) i)\right) \\
= & \left(\prod_{i=1}^{m-1}(1+r+(k-1) i)\right)+r \sum_{q=1}^{m-1}\left(\prod_{i=0}^{q-1}(r+(k-1) i)\right)\left(\prod_{i=q+1}^{m-1}(1+r+(k-1) i)\right) \\
= & \sum_{p=0}^{m-1}\left(\prod_{i=0}^{p-1}(r+(k-1) i)\right)\left(\prod_{i=p+1}^{m-1}(1+r+(k-1) i)\right) .
\end{aligned}
$$

Here we have used our induction hypothesis for $\Omega(k, m-2, r+k-1)$ in going from line 4 to line 5 in the above series of equalities.

Next we turn to general formulas for $B_{s, k n+j}^{(k)}, B_{0, s, k n+j}^{(k)}$ and $B_{1, s, k n+j}^{(k)}$ for all $k \geq 2$, $n \geq 0$, and $0 \leq j \leq k-1$. First, we develop formulas for the coefficients $B_{s, k n+j}^{(k)}$ by iterating the recursions (33) and (36) starting with our formula for $B_{n, k n+j}^{(k)}$ given in Theorem 10. That is, we shall prove the following theorem.

Theorem 13. For all $n \geq 0, k \geq 2$, and $0 \leq j \leq k-1$,

$$
\begin{aligned}
& B_{n-s, k n+j}^{(k)}= \\
& ((k-1) n+j) ! \sum_{r=0}^{s}(-1)^{s-r}\left(\begin{array}{c}
(k-1) n+j+r \\
r
\end{array}\right)\left(\begin{array}{c}
k n+j+1 \\
s-r
\end{array}\right) \prod_{i=0}^{n-1}(r+j+(k-1) i) .
\end{aligned}
$$

Proof. Our formula holds for $s=0$ by Theorem 10 .

Now assume that (39) holds for $s$ for all $n, k$, and $j$. Plugging $n-s$ for $s$ in (33), we obtain the recursion

$$
B_{n-s, k n+j+1}^{(k)}=(k n+j+1-s) B_{n-s, k n+j}^{(k)}+(s+1) B_{n-s-1, k n+j}^{(k)}
$$

or, equivalently,

$$
(s+1) B_{n-(s+1), k n+j}^{(k)}=B_{n-s, k n+j+1}^{(k)}-(k n+j+1-s) B_{n-s, k n+j}^{(k)}
$$

Thus by our induction hypothesis,

$$
\begin{aligned}
(s+1) & B_{n-(s+1), k n+j}^{(k)}=((k-1) n+j+1) ! \times \\
& \sum_{r=0}^{s}(-1)^{s-r}\left(\begin{array}{c}
(k-1) n+j+1+r \\
r
\end{array}\right)\left(\begin{array}{c}
k n+j+2 \\
s-r
\end{array}\right) \prod_{i=0}^{n-1}(r+j+1+(k-1) i) \\
& -(k n+j+1-s)((k-1) n+j) ! \times \\
& \sum_{r=0}^{s}(-1)^{s-r}\left(\begin{array}{c}
(k-1) n+j+r \\
r
\end{array}\right)\left(\begin{array}{c}
k n+j+1 \\
s-r
\end{array}\right) \prod_{i=0}^{n-1}(r+j+(k-1) i) .
\end{aligned}
$$


It follows that

$$
\begin{aligned}
& \frac{B_{n-(s+1), k n+j}^{(k)}}{((k-1) n+j) !}=\frac{(k-1) n+j+1}{s+1} \times \\
& \quad \sum_{r=0}^{s}(-1)^{s-r}\left(\begin{array}{c}
(k-1) n+j+1+r \\
r
\end{array}\right)\left(\begin{array}{c}
k n+j+2 \\
s-r
\end{array}\right) \prod_{i=0}^{n-1}(r+j+1+(k-1) i) \\
& \quad-\frac{k n+j+1-s}{s+1} \sum_{r=0}^{s}(-1)^{s-r}\left(\begin{array}{c}
(k-1) n+j+r \\
r
\end{array}\right)\left(\begin{array}{c}
k n+j+1 \\
s-r
\end{array}\right) \prod_{i=0}^{n-1}(r+j+(k-1) i) .
\end{aligned}
$$

We can then look at three terms. First the $r=s$ term on the first summand above is

$$
\begin{aligned}
\frac{(k-1) n+j+1}{s+1} & \left(\begin{array}{c}
(k-1) n+j+1+s \\
s
\end{array}\right) \prod_{i=0}^{n-1}(s+j+1+(k-1) i) \\
= & \left(\begin{array}{c}
(k-1) n+j+1+s \\
s+1
\end{array}\right) \prod_{i=0}^{n-1}(s+1+j+(k-1) i) .
\end{aligned}
$$

Second, the $r=0$ term from the second summand is

$$
(-1)^{s+1} \frac{k n+j+1-s}{s+1}\left(\begin{array}{c}
k n+j+1 \\
s
\end{array}\right) \prod_{i=0}^{n-1}(j+(k-1) i)=(-1)^{s+1}\left(\begin{array}{c}
k n+j+1 \\
s+1
\end{array}\right) \prod_{i=0}^{n-1}(j+(k-1) i) .
$$

Finally we can combine the terms involving $\prod_{i=0}^{n-1}(r+j+(k-1) i)$ from the first and second summands to obtain

$$
\begin{aligned}
& \sum_{r=1}^{s}(-1)^{s+1-r} \prod_{i=0}^{n-1}(r+j+(k-1) i) \times \\
& {\left[\frac{(k-1) n+j+1}{s+1}\left(\begin{array}{c}
(k-1) n+j+1+r-1 \\
r-1
\end{array}\right)\left(\begin{array}{c}
k n+j+2 \\
s-(r-1)
\end{array}\right)\right.} \\
& \left.+\frac{k n+j+1-s}{s+1}\left(\begin{array}{c}
(k-1) n+j+r \\
r
\end{array}\right)\left(\begin{array}{c}
k n+j+1 \\
s-r
\end{array}\right)\right]
\end{aligned}
$$

If we let $D_{r}$ be the factor in square brackets in the $r$-th term of (44), then we see that

$$
\begin{aligned}
D_{r}= & \frac{r}{r} \cdot \frac{(k-1) n+j+1}{s+1}\left(\begin{array}{c}
(k-1) n+j+r \\
r-1
\end{array}\right)\left(\begin{array}{c}
k n+j+2 \\
s+1-r
\end{array}\right) \\
& +\frac{s+1-r}{s+1-r} \cdot \frac{k n+j+1-s}{s+1}\left(\begin{array}{c}
(k-1) n+j+r \\
r
\end{array}\right)\left(\begin{array}{c}
k n+j+1 \\
s-r
\end{array}\right) \\
= & \frac{r}{s+1}\left(\begin{array}{c}
(k-1) n+j+r \\
r
\end{array}\right)\left(\begin{array}{c}
k n+j+2 \\
s+1-r
\end{array}\right) \\
& +\frac{s+1-r}{s+1-r} \cdot \frac{k n+j+1-s}{s+1}\left(\begin{array}{c}
(k-1) n+j+r \\
r
\end{array}\right)\left(\begin{array}{c}
k n+j+1 \\
s-r
\end{array}\right)
\end{aligned}
$$




$$
\begin{aligned}
& =\left(\begin{array}{c}
(k-1) n+j+r \\
r
\end{array}\right)\left[\frac{r}{s+1}\left(\begin{array}{c}
k n+j+2 \\
s+1-r
\end{array}\right)+\frac{s+1-r}{s+1-r} \cdot \frac{k n+j+1-s}{s+1}\left(\begin{array}{c}
k n+j+1 \\
s-r
\end{array}\right)\right] \\
& =\left(\begin{array}{c}
(k-1) n+j+r \\
r
\end{array}\right) \frac{(k n+j+1) \downarrow_{s-r}}{(s+1-r) !} \frac{1}{s+1}[r(k n+j+2)+(s+1-r)(k n+j+1-s)] \\
& =\left(\begin{array}{c}
(k-1) n+j+r \\
r
\end{array}\right) \frac{(k n+j+1) \downarrow s-r}{(s+1-r) !} \frac{1}{s+1}[(s+1)(k n+j+1-(s-r))] \\
& =\left(\begin{array}{c}
(k-1) n+j+r \\
r
\end{array}\right) \frac{(k n+j+1) \downarrow s+1-r}{(s+1-r) !} \\
& =\left(\begin{array}{c}
(k-1) n+j+r \\
r
\end{array}\right)\left(\begin{array}{c}
k n+j+1 \\
s+1-r
\end{array}\right) .
\end{aligned}
$$

Thus plugging in (45) into (44), we see that (44) is equal to

$$
\sum_{r=1}^{s}(-1)^{s+1-r}\left(\begin{array}{c}
(k-1) n+j+r \\
r
\end{array}\right)\left(\begin{array}{c}
k n+j+1 \\
s+1-r
\end{array}\right) \prod_{i=0}^{n-1}(r+j+(k-1) i) .
$$

Finally, combining (42), (43), and (46), we obtain that

$$
\begin{aligned}
& \frac{B_{n-(s+1), k n+j}^{(k)}}{((k-1) n+j) !} \\
& \quad=\sum_{r=0}^{s+1}(-1)^{s+1-r}\left(\begin{array}{c}
(k-1) n+j+r \\
r
\end{array}\right)\left(\begin{array}{c}
k n+j+1 \\
s+1-r
\end{array}\right) \prod_{i=0}^{n-1}(r+j+(k-1) i)
\end{aligned}
$$

as desired. Thus we have established our formula for $s+1$ and for all $n \geq 0, k \geq 2$, $j=0, \ldots, k-2$.

Next we substitute $n-s$ for $s$ in (36). We obtain the recursion that for $0 \leq j \leq k-2$,

$$
B_{n-s, k n+k}^{(k)}=(k n+k-s) B_{n-s, k n+k-1}^{(k)}+(s+1) B_{n-s-1, k n+k-1}^{(k)}
$$

or, equivalently,

$$
(s+1) B_{n-(s+1), k n+k-1}^{(k)}=B_{(n+1)-(s+1), k(n+1)}^{(k)}-(k n+k-s) B_{n-s, k n+k-1}^{(k)} .
$$

Given that we have already established our formula for $B_{n-s, k n+k-1}^{(k)}$ and using our induction hypothesis, we obtain that

$$
\begin{aligned}
& (s+1) B_{n-(s+1), k n+k-1}^{(k)}=((k-1)(n+1)) ! \times \\
& \sum_{r=0}^{s+1}(-1)^{s+1-r}\left(\begin{array}{c}
(k-1)(n+1)+r \\
r
\end{array}\right)\left(\begin{array}{c}
k(n+1)+1 \\
s+1-r
\end{array}\right) \prod_{i=0}^{n}(r+(k-1) i) \\
& -(k n+k-s)((k-1) n+k-1) ! \times \\
& \quad \sum_{r=0}^{s}(-1)^{s-r}\left(\begin{array}{c}
(k-1) n+k-1+r \\
r
\end{array}\right)\left(\begin{array}{c}
k n+k-1+1 \\
s-r
\end{array}\right) \prod_{i=0}^{n-1}(r+(k-1)+(k-1) i) .
\end{aligned}
$$


It follows that

$$
\begin{aligned}
& \frac{B_{n-(s+1), k n+k-1}^{(k)}}{((k-1) n+k-1) !} \\
& \quad=\frac{1}{s+1} \sum_{r=0}^{s+1}(-1)^{s+1-r}\left(\begin{array}{c}
(k-1) n+k-1+r \\
r
\end{array}\right)\left(\begin{array}{c}
k n+k+1 \\
s+1-r
\end{array}\right) r \prod_{i=1}^{n}(r+(k-1) i) \\
& \quad-\frac{k n+k-s}{s+1} \sum_{r=0}^{s}(-1)^{s-r}\left(\begin{array}{c}
(k-1) n+k-1+r \\
r
\end{array}\right)\left(\begin{array}{c}
k n+k \\
s-r
\end{array}\right) \prod_{i=0}^{n-1}(r+(k-1)+(k-1) i) .
\end{aligned}
$$

We can then look at three terms. First the $r=s+1$ term on the first summand of the final expression above is

$$
\begin{gathered}
\frac{1}{s+1}\left(\begin{array}{c}
(k-1) n+k-1+s+1 \\
s+1
\end{array}\right)(s+1) \prod_{i=0}^{n-1}(s+1+(k-1)+(k-1) i) \\
=\left(\begin{array}{c}
(k-1) n+k-1+s+1 \\
s+1
\end{array}\right) \prod_{i=0}^{n-1}(s+1+(k-1)+(k-1) i) .
\end{gathered}
$$

Second, the $r=0$ term from the second summand of the final expression for $\frac{B_{n-(s+1), k n+k-1}^{(k)}}{((k-1) n+k-1) !}$ above is

$$
(-1)^{s+1} \frac{k n+k-s}{s+1}\left(\begin{array}{c}
k n+k \\
s
\end{array}\right) \prod_{i=0}^{n-1}((k-1)+(k-1) i)=(-1)^{s+1}\left(\begin{array}{c}
k n+k \\
s+1
\end{array}\right) \prod_{i=0}^{n-1}((k-1)+(k-1) i) .
$$

Finally we can combine the terms involving $\prod_{i=0}^{n-1}(r+(k-1)+(k-1) i)$ from the first and second summands of the final expression for $\frac{B_{n-(s+1), k n+k-1}^{(k)}}{((k-1) n+k-1) !}$ above to obtain

$$
\begin{aligned}
& \sum_{r=1}^{s}(-1)^{s+1-r}\left(\begin{array}{c}
(k-1) n+k-1+r \\
r
\end{array}\right) \prod_{i=0}^{n-1}(r+(k-1)+(k-1) i) \times \\
& {\left[\frac{r}{s+1}\left(\begin{array}{c}
k n+k+1 \\
s+1-r
\end{array}\right)+\frac{k n+k-s}{s+1}\left(\begin{array}{c}
k n+k \\
s-r
\end{array}\right)\right] . }
\end{aligned}
$$

If we let $E_{r}$ be the factor in square brackets in the $r$-th term of (52), then we see that

$$
\begin{aligned}
E_{r} & =\frac{r}{s+1}\left(\begin{array}{c}
k n+k+1 \\
s+1-r
\end{array}\right)+\frac{s+1-r}{s+1-r} \cdot \frac{k n+k-s}{s+1}\left(\begin{array}{c}
k n+k \\
s-r
\end{array}\right) \\
& =\frac{(k n+k) \downarrow_{s-r}}{(s+1-r) !} \cdot \frac{1}{s+1}[r(k n+k+1)+(k n+k-s)(s+1-r)] \\
& =\frac{(k n+k) \downarrow_{s-r}}{(s+1-r) !} \cdot \frac{1}{s+1}[(s+1)(k n+k-(s-r))]
\end{aligned}
$$




$$
=\frac{(k n+k) \downarrow_{s+1-r}}{(s+1-r) !}=\left(\begin{array}{c}
k n+k \\
s+1-r
\end{array}\right) .
$$

Thus plugging in (53) into (52), we see that (52) is equal to

$$
\sum_{r=1}^{s}(-1)^{s+1-r}\left(\begin{array}{c}
(k-1) n+k-1+r \\
r
\end{array}\right)\left(\begin{array}{c}
k n+k \\
s+1-r
\end{array}\right) \prod_{i=0}^{n-1}(r+(k-1)+(k-1) i) .
$$

Finally, combining (50), (51), and (54), we obtain that

$$
\begin{aligned}
& \frac{B_{n-(s+1), k n+k-1}^{(k)}}{((k-1) n+k-1) !}= \\
& \sum_{r=0}^{s+1}(-1)^{s+1-r}\left(\begin{array}{c}
(k-1) n+k-1+r \\
r
\end{array}\right)\left(\begin{array}{c}
k n+k \\
s+1-r
\end{array}\right) \prod_{i=0}^{n-1}(r+(k-1)+(k-1) i)
\end{aligned}
$$

as desired.

As a corollary to Theorem 13 we get combinatorial proofs for two special cases of the Saalschütz's identity. Recall that in terms of generalized hypergeometric functions it can be written as

$$
{ }_{3} F_{2}\left[\begin{array}{ccc}
a & b & c \\
d & e & ; 1
\end{array}\right]=\frac{(d-a)_{|c|}(d-b)_{|c|}}{d_{|c|}(d-a-b)_{|c|}}
$$

where $d+e=a+b+c+1$ and $c$ is a negative integer ${ }^{2}$.

Corollary 3. The following identities hold:

$$
\begin{gathered}
\frac{n+1}{s+1}\left(\begin{array}{c}
n \\
s
\end{array}\right)^{2}=\sum_{r=0}^{n-s}(-1)^{n-s-r}\left(\begin{array}{c}
n+r \\
r
\end{array}\right)\left(\begin{array}{c}
n+r+1 \\
r
\end{array}\right)\left(\begin{array}{c}
2 n+2 \\
n-s-r
\end{array}\right) \\
\left(\begin{array}{c}
n-1 \\
s
\end{array}\right)\left(\begin{array}{c}
n+1 \\
s+1
\end{array}\right)=\sum_{r=0}^{n-s}(-1)^{n-s-r}\left(\begin{array}{c}
n+r \\
r
\end{array}\right)\left(\begin{array}{c}
n+r-1 \\
r-1
\end{array}\right)\left(\begin{array}{c}
2 n+1 \\
n-s-r
\end{array}\right) .
\end{gathered}
$$

Proof. The RHS of the first identity is $B_{s, 2 n+1}^{(2)} /(n !(n+1) !)$ (we use Theorem 13 for $k=2$, $j=1$ and $s=n-s)$. However, $B_{s, 2 n+1}^{(2)}$ counts exactly the same objects as $P_{s, 2 n+1}=$ $P_{0, s, 2 n+1}+P_{1, s, 2 n+1}=\frac{1}{s+1}\left(\begin{array}{c}n \\ s\end{array}\right)^{2}((n+1) !)^{2}$ does in [6]. The RHS of the second identity is $B_{s, 2 n}^{(2)} /(n !)^{2}$, and $B_{s, 2 n}^{(2)}$ is the same as $P_{s, 2 n}=P_{0, s, 2 n}+P_{1, s, 2 n}=(n !)^{2}\left(\begin{array}{c}n-1 \\ s\end{array}\right)\left(\begin{array}{c}n+1 \\ s+1\end{array}\right)$ in $[6]$.

Next we prove formulas for the coefficients $B_{1, s, k n+j}^{(k)}$ by iterating the recursions (32) and (35) starting from our formula for $B_{1, n-1, k n+j}^{(k)}$ given in Theorem 11.

\footnotetext{
${ }^{2}$ For the first identity in Corollary $3, a=n+2, b=n+1, c=s-n, d=1$, and $e=n+s+3$; for the second identity there, $a=n+1, b=n, c=s-n, d=0$, and $e=n+s+2$.
} 
Theorem 14. For all $n \geq 0, k \geq 2$, and $0 \leq j \leq k-1$,

$$
B_{1, n-1-s, k n+j}^{(k)}=((k-1) n+j) ! \sum_{r=0}^{s}(-1)^{s-r}\left(\begin{array}{c}
(k-1) n+j+r \\
r
\end{array}\right)\left(\begin{array}{c}
k n+j \\
s-r
\end{array}\right) \Omega(k, n, r+j) .
$$

where $\Omega(k, n, r)$ is given by (38).

Proof. Our formula holds for $s=0$ by Theorem 11 .

Now assume that(55) holds for $s$ for all $n, k$, and $j$. Plugging $n-s-1$ for $s$ in (32), we obtain the recursion that for $0 \leq j \leq k-2$,

$$
B_{1, n-1-s, k n+j+1}^{(k)}=(k n+j-s) B_{1, n-1-s, k n+j}^{(k)}+(s+1) B_{1, n-1-s-1, k n+j}^{(k)}
$$

or, equivalently,

$$
(s+1) B_{1, n-1-(s+1), k n+j}^{(k)}=B_{1, n-1-s, k n+j+1}^{(k)}-(k n+j-s) B_{1, n-1-s, k n+j}^{(k)} .
$$

Thus by our induction hypothesis,

$$
\begin{aligned}
& (s+1) B_{1, n-1-(s+1), k n+j}^{(k)}= \\
& ((k-1) n+j+1) ! \sum_{r=0}^{s}(-1)^{s-r}\left(\begin{array}{c}
(k-1) n+j+1+r \\
r
\end{array}\right)\left(\begin{array}{c}
k n+j+1 \\
s-r
\end{array}\right) \Omega(k, n, r+j+1) \\
& -(k n+j-s)((k-1) n+j) ! \sum_{r=0}^{s}(-1)^{s-r}\left(\begin{array}{c}
(k-1) n+j+r \\
r
\end{array}\right)\left(\begin{array}{c}
k n+j \\
s-r
\end{array}\right) \Omega(k, n, r+j) .
\end{aligned}
$$

It follows that

$$
\begin{aligned}
& \frac{B_{1, n-1-(s+1), k n+j}^{(k)}}{((k-1) n+j) !} \\
& =\frac{(k-1) n+j+1}{s+1} \sum_{r=0}^{s}(-1)^{s-r}\left(\begin{array}{c}
(k-1) n+j+1+r \\
r
\end{array}\right)\left(\begin{array}{c}
k n+j+1 \\
s-r
\end{array}\right) \Omega(k, n, r+j+1) \\
& \quad-\frac{k n+j-s}{s+1} \sum_{r=0}^{s}(-1)^{s-r}\left(\begin{array}{c}
(k-1) n+j+r \\
r
\end{array}\right)\left(\begin{array}{c}
k n+j \\
s-r
\end{array}\right) \Omega(k, n, r+j) .
\end{aligned}
$$

We can then look at three terms. First the $r=s$ term on the first summand above is

$$
\begin{aligned}
& \frac{(k-1) n+j+1}{s+1}\left(\begin{array}{c}
(k-1) n+j+1+s \\
s
\end{array}\right) \Omega(k, n, s+j+1) \\
& =\left(\begin{array}{c}
(k-1) n+j+1+s \\
s+1
\end{array}\right) \Omega(k, n, s+j+1) .
\end{aligned}
$$


Second, the $r=0$ term from the second summand is

$$
(-1)^{s+1} \frac{k n+j-s}{s+1}\left(\begin{array}{c}
k n+j \\
s
\end{array}\right) \Omega(k, n, j)=(-1)^{s+1}\left(\begin{array}{c}
k n+j \\
s+1
\end{array}\right) \Omega(k, n, j) .
$$

Finally we can combine the terms involving $\Omega(k, n, r+j)$ from the first and second summands to obtain

$$
\begin{aligned}
& \sum_{r=1}^{s}(-1)^{s+1-r} \Omega(k, n, r+j) \times \\
& {\left[\begin{array}{c}
(k-1) n+j+1 \\
s+1
\end{array}\left(\begin{array}{c}
(k-1) n+j+1+r-1 \\
r-1
\end{array}\right)\left(\begin{array}{c}
k n+j+1 \\
s-(r-1)
\end{array}\right)\right.} \\
& \left.+\frac{k n+j-s}{s+1}\left(\begin{array}{c}
(k-1) n+j+r \\
r
\end{array}\right)\left(\begin{array}{c}
k n+j \\
s-r
\end{array}\right)\right] .
\end{aligned}
$$

If we let $F_{r}$ be the factor in square brackets in the $r$-th term of (60), then we see that

$$
\begin{aligned}
F_{r}= & \frac{r}{r} \cdot \frac{(k-1) n+j+1}{s+1}\left(\begin{array}{c}
(k-1) n+j+r \\
r-1
\end{array}\right)\left(\begin{array}{c}
k n+j+1 \\
s+1-r
\end{array}\right) \\
& +\frac{s+1-r}{s+1-r} \cdot \frac{k n+j-s}{s+1}\left(\begin{array}{c}
(k-1) n+j+r \\
r
\end{array}\right)\left(\begin{array}{c}
k n+j \\
s-r
\end{array}\right) \\
= & \frac{r}{s+1}\left(\begin{array}{c}
(k-1) n+j+r \\
r
\end{array}\right)\left(\begin{array}{c}
k n+j+1 \\
s+1-r
\end{array}\right) \\
& +\frac{s+1-r}{s+1-r} \cdot \frac{k n+j-s}{s+1}\left(\begin{array}{c}
(k-1) n+j+r \\
r
\end{array}\right)\left(\begin{array}{c}
k n+j \\
s-r
\end{array}\right) \\
= & \left(\begin{array}{c}
(k-1) n+j+r \\
r
\end{array}\right)\left[\frac{r}{s+1}\left(\begin{array}{c}
k n+j+1 \\
s+1-r
\end{array}\right)+\frac{s+1-r}{s+1-r} \cdot \frac{k n+j-s}{s+1}\left(\begin{array}{c}
k n+j \\
s-r
\end{array}\right)\right] \\
= & \left(\begin{array}{c}
(k-1) n+j+r \\
r
\end{array}\right) \frac{(k n+j) \downarrow s-r}{(s+1-r) !} \cdot \frac{1}{s+1}[r(k n+j+1)+(s+1-r)(k n+j-s)] \\
= & \left(\begin{array}{c}
(k-1) n+j+r \\
r
\end{array}\right) \frac{(k n+j) \downarrow s-r}{(s+1-r) !} \cdot \frac{1}{s+1}[(s+1)(k n+j-(s-r))] \\
= & \left(\begin{array}{c}
(k-1) n+j+r \\
r
\end{array}\right) \frac{(k n+j) \downarrow s+1-r}{(s+1-r) !} \\
= & \left(\begin{array}{c}
(k-1) n+j+r \\
r
\end{array}\right)\left(\begin{array}{c}
k n+j \\
s+1-r
\end{array}\right) .
\end{aligned}
$$

Thus plugging in (61) into (60), we see that (60) is equal to

$$
\sum_{r=1}^{s}(-1)^{s+1-r}\left(\begin{array}{c}
(k-1) n+j+r \\
r
\end{array}\right)\left(\begin{array}{c}
k n+j \\
s+1-r
\end{array}\right) \Omega(k, n, r+j) .
$$

Finally, combining (58), (59), and (62), we obtain that

$$
\frac{B_{1, n-1-(s+1), k n+j}^{(k)}}{((k-1) n+j) !}=\sum_{r=0}^{s+1}(-1)^{s+1-r}\left(\begin{array}{c}
(k-1) n+j+r \\
r
\end{array}\right)\left(\begin{array}{c}
k n+j \\
s+1-r
\end{array}\right) \Omega(k, n, r+j)
$$


as desired. Thus we have established our formula for all $n \geq 0, k \geq 2, j=0, \ldots, k-2$ for $s+1$.

Next if we substitute $n-s-1$ for $s$ in (35), we obtain the recursion

$$
\begin{aligned}
& B_{1, n-1-s, k n+k}^{(k)}= \\
& (k n+k-1-s) B_{1, n-1-s, k n+k-1}^{(k)}+(s+2) B_{1, n-1-s-1, k n+k-1}^{(k)}+B_{0, n-1-s, k n+k-1}^{(k)}
\end{aligned}
$$

or, equivalently,

$$
\begin{aligned}
(s+2) & B_{1, n-1-(s+1), k n+k-1}^{(k)} \\
= & B_{1,(n+1)-1-(s+1), k(n+1)}^{(k)}-(k n+k-1-s) B_{1, n-1-s, k n+k-1}^{(k)}-B_{0, n-1-s, k n+k-1}^{(k)} \\
= & B_{1,(n+1)-1-(s+1), k(n+1)}^{(k)}-(k n+k-1-s) B_{1, n-1-s, k n+k-1}^{(k)} \\
& \quad-\left(B_{n-(s+1), k n+k-1}^{(k)}-B_{1, n-1-s, k n+k-1}^{(k)}\right) \\
= & B_{1,(n+1)-1-(s+1), k(n+1)}^{(k)}-(k n+k-s-2) B_{1, n-1-s, k n+k-1}^{(k)}-B_{n-(s+1), k n+k-1}^{(k)} .
\end{aligned}
$$

Given that we have already got our formula for $B_{n-(s+1), k n+k-1}^{(k)}$ and $B_{1, n-1-s, k n+k-1}^{(k)}$, we use our induction hypothesis to obtain that

$$
\begin{aligned}
(s+2) & B_{1, n-1-(s+1), k n+k-1}^{(k)} \\
= & ((k-1)(n+1)) ! \sum_{r=0}^{s+1}(-1)^{s+1-r}\left(\begin{array}{c}
k-1)(n+1)+r \\
r
\end{array}\right)\left(\begin{array}{c}
k(n+1) \\
s+1-r
\end{array}\right) \Omega(k, n+1, r) \\
& -(k n+k-s-2)((k-1) n+k-1) ! \times \\
& \sum_{r=0}^{s}(-1)^{s-r}\left(\begin{array}{c}
(k-1) n+k-1+r \\
r
\end{array}\right)\left(\begin{array}{c}
k n+k-1 \\
s-r
\end{array}\right) \Omega(k, n, r+k-1) \\
& -((k-1) n+k-1) ! \times \\
& \sum_{r=0}^{s+1}(-1)^{s+1-r}\left(\begin{array}{c}
(k-1)(n+1)+r \\
r
\end{array}\right)\left(\begin{array}{c}
k n+k \\
s+1-r
\end{array}\right) \prod_{i=0}^{n-1}(r+(k-1)+(k-1) i) .
\end{aligned}
$$

It follows that

$$
\begin{aligned}
& \frac{B_{1, n-1-(s+1), k n+k-1}^{(k)}}{((k-1) n+k-1) !} \\
& =\frac{1}{s+2} \sum_{r=0}^{s+1}(-1)^{s+1-r}\left(\begin{array}{c}
(k-1) n+k-1+r \\
r
\end{array}\right)\left(\begin{array}{c}
k n+k \\
s+1-r
\end{array}\right) \times \\
& {\left[\Omega(k, n+1, r)-\prod_{i=0}^{n-1}(r+(k-1)+(k-1) i)\right]} \\
& \quad-\frac{k n+k-s-2}{s+2} \sum_{r=0}^{s}(-1)^{s-r}\left(\begin{array}{c}
(k-1) n+k-1+r \\
r
\end{array}\right)\left(\begin{array}{c}
k n+k-1 \\
s-r
\end{array}\right) \Omega(k, n, r+k-1) .
\end{aligned}
$$


Next we shall rewrite the term $\left[\Omega(k, n+1, r)-\prod_{i=0}^{n-1}(r+(k-1)+(k-1) i)\right]$. Note that

$$
\begin{aligned}
& \Omega(k, n+1, r)-\prod_{i=0}^{n-1}(r+(k-1)+(k-1) i) \\
&=\sum_{p=0}^{n}\left(\prod_{i=0}^{p-1}(r+(k-1) i)\right)\left(\prod_{i=p+1}^{n}(1+r+(k-1) i)\right) \\
& \quad-\prod_{i=0}^{n-1}(r+(k-1)+(k-1) i) \\
&=\sum_{p=1}^{n} r\left(\prod_{i=1}^{p-1}(r+(k-1) i)\right)\left(\prod_{i=p+1}^{n}(1+r+(k-1) i)\right) \\
& \quad+\prod_{i=1}^{n}(1+r+(k-1) i)-\prod_{i=0}^{n-1}(r+(k-1)+(k-1) i) \\
&=\quad r \sum_{p=0}^{n-1}\left(\prod_{i=0}^{p-1}(r+(k-1)+(k-1) i)\right)\left(\prod_{i=p+1}^{n-1}(1+r+(k-1)+(k-1) i)\right) \\
& \quad+\prod_{i=0}^{n-1}(1+r+(k-1)+(k-1) i)-\prod_{i=0}^{n-1}(r+(k-1)+(k-1) i) \\
&= r \Omega(k, n, r+k-1)+\prod_{i=0}^{n-1}(1+r+(k-1)+(k-1) i)-\prod_{i=0}^{n-1}(r+(k-1)+(k-1) i) \\
& r(k, n, r+k-1)+\Omega(k, n, r+k-1)=(1+r) \Omega(k, n+k-1) .
\end{aligned}
$$

Here on the next to last line, we have used Lemma 12. Thus plugging (66) into our expression for $\frac{B_{1, n-1-(s+1), k n+k-1}^{(k)}}{((k-1) n+k-1) !}$, we obtain that

$$
\begin{aligned}
& \frac{B_{1, n-1-(s+1), k n+k-1}^{(k)}}{((k-1) n+k-1) !} \\
& \quad=\frac{1}{s+2} \sum_{r=0}^{s+1}(-1)^{s+1-r}\left(\begin{array}{c}
(k-1) n+k-1+r \\
r
\end{array}\right)\left(\begin{array}{c}
k n+k \\
s+1-r
\end{array}\right)(1+r) \Omega(k, n, r+k-1) \\
& \quad-\frac{k n+k-s-2}{s+2} \sum_{r=0}^{s}(-1)^{s-r}\left(\begin{array}{c}
(k-1) n+k-1+r \\
r
\end{array}\right)\left(\begin{array}{c}
k n+k-1 \\
s-r
\end{array}\right) \Omega(k, n, r+k-1) .
\end{aligned}
$$

We can then look at two terms. First, the $r=s+1$ term on the first summand of the final expression above is 


$$
\begin{array}{r}
\frac{1}{s+2}\left(\begin{array}{r}
(k-1) n+k-1+s+1 \\
s+1
\end{array}\right)(s+2) \Omega(k, n, s+1+k-1) \\
=\left(\begin{array}{c}
(k-1) n+k+s \\
s+1
\end{array}\right) \Omega(k, n, s+k) .
\end{array}
$$

Second, we can combine the terms involving $\Omega(k, n, r+k-1)$ from the first and second summands of the final expression for $\frac{B_{1, n-1-(s+1), k n+k-1}^{(k)}}{((k-1) n+k-1) !}$ above to obtain

$$
\begin{aligned}
& \sum_{r=0}^{s}(-1)^{s+1-r}\left(\begin{array}{c}
(k-1) n+k-1+r \\
r
\end{array}\right) \Omega(k, n, r+k-1) \times \\
& {\left[\frac{1+r}{s+2}\left(\begin{array}{c}
k n+k \\
s+1-r
\end{array}\right)+\frac{k n+k-s-2}{s+2}\left(\begin{array}{c}
k n+k-1 \\
s-r
\end{array}\right)\right] .}
\end{aligned}
$$

If we let $G_{r}$ be the factor in square brackets in the $r$-th term of (68), then we see that

$$
\begin{aligned}
G_{r} & =\frac{1+r}{s+2}\left(\begin{array}{c}
k n+k \\
s+1-r
\end{array}\right)+\frac{s+1-r}{s+1-r} \cdot \frac{k n+k-s-2}{s+2}\left(\begin{array}{c}
k n+k-1 \\
s-r
\end{array}\right) \\
& =\frac{(k n+k-1) \downarrow_{s-r}}{(s+1-r) !} \frac{1}{s+2}[(1+r)(k n+k)+(k n+k-(s+2))(s+1-r)] \\
& =\frac{(k n+k-1) \downarrow_{s-r}}{(s+1-r) !} \frac{1}{s+2}[(s+2)(k n+k-1-(s-r))] \\
& =\frac{(k n+k-1) \downarrow_{s+1-r}}{(s+1-r) !} \\
& =\left(\begin{array}{c}
k n+k-1 \\
s+1-r
\end{array}\right) .
\end{aligned}
$$

Thus plugging in (69) into (68), we see that (68) is equal to

$$
\sum_{r=0}^{s}(-1)^{s+1-r}\left(\begin{array}{c}
(k-1) n+k-1+r \\
r
\end{array}\right)\left(\begin{array}{c}
k n+k-1 \\
s+1-r
\end{array}\right) \Omega(k, n, r+k-1) .
$$

Finally, combining (67) and (70), we obtain that

$$
\frac{B_{1, n-1-(s+1), k n+k-1}^{(k)}}{((k-1) n+k-1) !}=\sum_{r=0}^{s+1}(-1)^{s+1-r}\left(\begin{array}{c}
(k-1) n+k-1+r \\
r
\end{array}\right)\left(\begin{array}{c}
k n+k-1 \\
s+1-r
\end{array}\right) \Omega(k, n, r+k-1)
$$

as desired.

Note that it follows that for all $n \geq 0, k \geq 2,0 \leq j \leq k-1$, and $0 \leq s \leq n-1$,

$$
B_{0, n-1-s, k n+j}^{(k)}=B_{n-(s+1), k n+j}^{(k)}-B_{1, n-1-s, k n+j}^{(k)}
$$




$$
\begin{aligned}
= & ((k-1) n+j) ! \sum_{r=0}^{s+1}(-1)^{s+1-r}\left(\begin{array}{c}
(k-1) n+j+r \\
r
\end{array}\right)\left(\begin{array}{c}
k n+j+1 \\
s+1-r
\end{array}\right) \prod_{i=0}^{n-1}(r+j+(k-1) i) \\
& -((k-1) n+j) ! \sum_{r=0}^{s}(-1)^{s-r}\left(\begin{array}{c}
(k-1) n+j+r \\
r
\end{array}\right)\left(\begin{array}{c}
k n+j \\
s-r
\end{array}\right) \Omega(k, n, r+j) .
\end{aligned}
$$

Thus

$$
\begin{aligned}
& \frac{B_{0, n-(s+1), k n+j}^{(k)}}{((k-1) n+j) !}=\left(\begin{array}{c}
(k-1) n+j+s+1 \\
s+1
\end{array}\right) \prod_{i=0}^{n-1}(s+1+j+(k-1) i) \\
& +\sum_{r=0}^{s}(-1)^{s+1-r}\left(\begin{array}{c}
(k-1) n+j+r \\
r
\end{array}\right)\left(\begin{array}{c}
k n+j+1 \\
s+1-r
\end{array}\right) \prod_{i=0}^{n-1}(r+j+(k-1) i) \\
& -\sum_{r=0}^{s}(-1)^{s-r}\left(\begin{array}{c}
(k-1) n+j+r \\
r
\end{array}\right)\left(\begin{array}{c}
k n+j \\
s-r
\end{array}\right) \times \\
& {\left[\prod_{i=0}^{n-1}(1+r+j+(k-1) i)-\prod_{i=0}^{n-1}(r+j+(k-1) i)\right]} \\
& =\left(\begin{array}{c}
(k-1) n+j+s+1 \\
s+1
\end{array}\right) \prod_{i=0}^{n-1}(s+1+j+(k-1) i) \\
& +\sum_{r=0}^{s}(-1)^{s+1-r}\left(\begin{array}{c}
(k-1) n+j+r \\
r
\end{array}\right)\left[\left(\begin{array}{c}
k n+j+1 \\
s+1-r
\end{array}\right)-\left(\begin{array}{c}
k n+j \\
s-r
\end{array}\right)\right] \prod_{i=0}^{n-1}(r+j+(k-1) i) \\
& -\sum_{r=0}^{s}(-1)^{s-r}\left(\begin{array}{c}
(k-1) n+j+r \\
r
\end{array}\right)\left(\begin{array}{c}
k n+j \\
s-r
\end{array}\right) \prod_{i=0}^{n-1}(1+r+j+(k-1) i) \\
& =\left(\begin{array}{c}
(k-1) n+j+s+1 \\
s+1
\end{array}\right) \prod_{i=0}^{n-1}(s+1+j+(k-1) i) \\
& +\sum_{r=0}^{s}(-1)^{s+1-r}\left(\begin{array}{c}
(k-1) n+j+r \\
r
\end{array}\right)\left(\begin{array}{c}
k n+j \\
s+1-r
\end{array}\right) \prod_{i=0}^{n-1}(r+j+(k-1) i) \\
& -\sum_{r=0}^{s}(-1)^{s-r}\left(\begin{array}{c}
(k-1) n+k-1+r \\
r
\end{array}\right)\left(\begin{array}{c}
k n+j \\
s-r
\end{array}\right) \prod_{i=0}^{n-1}(1+r+j+(k-1) i) \\
& =\sum_{r=0}^{s+1}(-1)^{s+1-r}\left(\begin{array}{c}
(k-1) n+j+r \\
r
\end{array}\right)\left(\begin{array}{c}
k n+j \\
s+1-r
\end{array}\right) \prod_{i=0}^{n-1}(r+j+(k-1) i) \\
& -\sum_{r=0}^{s}(-1)^{s-r}\left(\begin{array}{c}
(k-1) n+k-1+r \\
r
\end{array}\right)\left(\begin{array}{c}
k n+j \\
s-r
\end{array}\right) \prod_{i=0}^{n-1}(1+r+j+(k-1) i) .
\end{aligned}
$$

Thus we have proved the following.

Theorem 15. For all $n \geq 0, k \geq 2$, and $0 \leq j \leq k-1$, 
1. $B_{0, n, k n+j}^{(k)}=((k-1) n+j) ! \prod_{i=0}^{n-1}(j+(k-1) i)$ and

2. $B_{0, n-1-s, k n+j}^{(k)}=$

$$
\begin{aligned}
& \sum_{r=0}^{s+1}(-1)^{s+1-r}\left(\begin{array}{c}
(k-1) n+j+r \\
r
\end{array}\right)\left(\begin{array}{c}
k n+j \\
s+1-r
\end{array}\right) \prod_{i=0}^{n-1}(r+j+(k-1) i) \\
& -\sum_{r=0}^{s}(-1)^{s-r}\left(\begin{array}{c}
(k-1) n+k-1+r \\
r
\end{array}\right)\left(\begin{array}{c}
k n+j \\
s-r
\end{array}\right) \prod_{i=0}^{n-1}(1+r+j+(k-1) i) \text { for } 0 \leq s \leq n-1 .
\end{aligned}
$$

We can get another set of formulas for the coefficients $B_{s, k n+j}^{(k)}$ for all $k \geq 2, n \geq 0$ by iterating the recursions (33) and (36) starting with our formula for $B_{0, k n+j}^{(k)}$ given in Theorem 9. The proof is very similar to the proofs of our previous theorems so that we shall omit the details.

Theorem 16. For all $k \geq 2, n \geq 0,0 \leq j \leq k-1$, and $0 \leq s \leq n$,

$$
\begin{aligned}
& B_{s, k n+j}^{(k)}= \\
& ((k-1) n+j) !\left[\sum_{r=0}^{s}(-1)^{s-r}\left(\begin{array}{c}
(k-1) n+j+r \\
r
\end{array}\right)\left(\begin{array}{c}
k n+j+1 \\
s-r
\end{array}\right) \prod_{i=1}^{n}(1+r+(k-1) i)\right] .
\end{aligned}
$$

Once again, there are a number of identities that now follow. For example, it follows from Theorems 13 and 16 that

Theorem 17. For all $n \geq 0, k \geq 2$, and $0 \leq j \leq k-1$,

$$
\begin{aligned}
& \sum_{r=0}^{s}(-1)^{s-r}\left(\begin{array}{c}
(k-1) n+j+r \\
r
\end{array}\right)\left(\begin{array}{c}
k n+j+1 \\
s-r
\end{array}\right) \prod_{i=1}^{n}(1+r+(k-1) i) \\
& =\sum_{r=0}^{n-s}(-1)^{n-s-r}\left(\begin{array}{c}
(k-1) n+j+r \\
r
\end{array}\right)\left(\begin{array}{c}
k n+j+1 \\
n-s-r
\end{array}\right) \prod_{i=0}^{n-1}(r+j+(k-1) i) .
\end{aligned}
$$

\section{Bijective proofs related to the context}

In this section we generalize bijective proofs from [6] to show some relations for the coefficients of $A_{n}^{(k)}(x)$ and $B_{n}^{(k)}(x, z)$ for certain $n$. An important observation here is that the complement $\pi^{c}$ for a $(k n+k-1)$-permutation $\pi$ has the following property: $\pi_{i}$ is divisible by $k$ if and only if $\pi_{i}^{c}$ is divisible by $k$ for $i=1,2, \ldots, k n+k-1$.

First it is easy to see that the polynomials $A_{k n+k-1}^{(k)}(x)$ and $B_{k n+k-1}^{(k)}(x, z)$ have simple symmetry properties.

Theorem 18. For all $k \geq 2$ and $n \geq 0$

$$
\begin{aligned}
A_{s, k n+k-1}^{(k)} & =A_{n-s, k n+k-1}^{(k)} \text { for } 0 \leq s \leq n \\
B_{0, s, k n+k-1}^{(k)} & =B_{0, n-s, k n+k-1}^{(k)} \text { for } 0 \leq s \leq n, \text { and } \\
B_{1, s, k n+k-1}^{(k)} & =B_{1, n-1-s, k n+k-1}^{(k)} \text { for } 0 \leq s \leq n-1 .
\end{aligned}
$$


Proof. The map which sends $\sigma$ to $\sigma^{c}$ gives a bijective proof of all three results.

Lemma 19. For all $n \geq 0, k \geq 2$, and $0 \leq s \leq n$,

$$
A_{s, k n+k-1}^{(k)}=B_{s, k n+k-1}^{(k)} \text {. }
$$

Proof. Given $\sigma=\sigma_{1} \cdots \sigma_{k n+k-1} \in S_{k n+k-1}$, let $\sigma^{*}=\left(k n+k-\sigma_{k n+k-1}\right) \cdots\left(k n+k-\sigma_{1}\right)$ be the permutation that results by taking the complement of $\sigma$ and then the reversing the result. It is then easy to see that $\overleftarrow{d e s}_{k N}(\sigma)=\overrightarrow{d e s}_{k N}\left(\sigma^{*}\right)$ which shows that $A_{s, k n+k-1}^{(k)}=$ $B_{s, k n+k-1}^{(k)}$.

Theorem 20. For all $k \geq 3, n \geq 0$, and $1 \leq j \leq\lfloor n / k\rfloor$,

$$
A_{j, k n+k-2}^{(k)}(x)=A_{n-j, k n+k-2}^{(k)}(x) .
$$

Thus $A_{k n+k-2}^{(k)}(x)$ is symmetric for $n \geq 0$ and $k \geq 3$.

Proof. Given a permutation $\sigma=\sigma_{1} \cdots \sigma_{k n+k-2} \in \mathcal{S}_{k n+k-2}$ with $\overleftarrow{\mathrm{des}}_{E}(\sigma)=j$, apply the complement to the permutation $\sigma^{\prime}=\sigma(k n+k-1)$, that is, $\sigma^{\prime}$ is obtained from $\sigma$ by adding a dummy letter $(k n+k-1)$ at the end. In the obtained permutation $\sigma^{\prime c}$, make a cyclic shift to the left to make the letter $(k n+k-1)$ be the first one. Remove $(k n+k-1)$ to get a $(k n+k-2)$-permutation $\sigma^{*}$ with $\overleftarrow{d e s}_{k N}\left(\sigma^{*}\right)=n-j$. To reverse this procedure adjoin $(k n+k-1)$ from the left to a given permutation $\sigma^{*}$ with $\overleftarrow{d e s}_{k N}\left(\sigma^{*}\right)=n-j$. Then make a cyclic shift to the right to make 1 be the rightmost letter. Use the complement and remove $(k n+k-1)$ from the obtained permutation to get a permutation $\sigma$ with $\overleftarrow{\operatorname{des}}_{E}(\sigma)=j$.

The map described above and its reverse are clearly injective. We only need to justify that given $j$ occurrences of the descents in $\sigma$, we get $(n-j)$ occurrences in $\sigma^{*}$ (the reverse to this statement will follow using the same arguments). Notice that adding $(k n+k-1)$ at the end does not increase the number of descents. Since $\sigma^{\prime}$ ends with a number which is not equivalent to $k \bmod 0, \sigma^{\prime c}$ has $n-j$ descents. If $\sigma^{\prime}=A 1 B(k n+k-1)$, where $A$ and $B$ are some factors, then $\sigma^{c}=A^{c}(k n+k-1) B^{c} 1$ and $\sigma^{*}$ is $(k n+k-1) B^{c} 1 A^{c}$ without $(k n+k-1)$. The last thing to observe is that moving $A^{c}$ to the end of $\sigma^{c}$ does not create a new descent since it cannot start with 1 , also we do not lose any descents since none of them can end with $(k n+k-1)$. So, $\sigma^{*}$ has $n-k$ descents.

For instance, the permutation 38215647 counted by $A_{3,8}^{(2)}(k=2, j=3$, and $n=4)$ is mapped to the permutation counted by $A_{1,8}^{(2)}$ through the following steps:

$$
38215647 \rightarrow 382156479 \rightarrow 728954631 \rightarrow 954631728 \rightarrow 54631728 .
$$

Theorem 21. For all $k \geq 3, n \geq 0$, and $1 \leq j \leq\lfloor n / k\rfloor$,

$$
A_{j, k n+k-2}^{(k)}=B_{0, j, k n+k-2}^{(k)}+B_{1, j-1, k n+k-2}^{(k)} .
$$


Proof. We now keep track of whether or not this is a number divisible by $k$ to the left of 1 in $\sigma=\sigma_{1} \sigma_{2} \cdots \sigma_{k n+k-2} \in \mathcal{S}_{k n+k-2}$ with $\overleftarrow{d e s}_{k N}(\sigma)=j$. We do not provide all the justifications in our explanation of the map, since they are similar to those in the proof of Theorem 20.

Suppose $\sigma^{\prime}=\sigma(k n+k-1)=A x 1 B(k n+k-1)$, where $A$ and $B$ are some factors and $x$ is a number. Apply the complement and reverse to $\sigma^{\prime}$ to get $\left(\sigma^{\prime}\right)^{c r}=1 B^{c r}(k n+k-1) x^{c} A^{c r}$. Make a cyclic shift to the left in $\left(\sigma^{\prime}\right)^{c r}$ to make the number $(k n+k-1)$ be the first one and to get $\sigma^{*}=(k n+k-1) x^{c} A^{c r} 1 B^{c r}$. One can check that $\overleftarrow{d e s}_{k N}(\sigma)=\overrightarrow{\operatorname{des}}_{k N}\left(\sigma^{*}\right)=j$. Also, $x$ is divisible by $k$ if and only if $x^{c}$ is divisible by $k$. Now, if we remove $(k n+k-1)$ from $\sigma^{*}$ and $x^{c}$ is $0 \bmod k$, we loose one descent obtaining a permutation counted by $B_{1, j-1, k n+k-2}^{(k)}$; if we remove $(k n+k-1)$ from $\sigma^{*}$ and $x^{c}$ is not o $\bmod k$, the number of descents in the obtained permutation is the same, $j$, and thus we get a permutation counted by $B_{0, j, k n+k-2}^{(k)}$. Note that if $A x$ is the empty word, that is, $\sigma$ starts with 1 , then this case is treated as the case " $x$ is not $0 \bmod k$ " since $\sigma^{*}$ will start with $(k n+k-1) 1$. Thus one may think of $k n+k-1$ as the (cyclic) predecessor of 1 in this case, that is, $x=k n+k-1$.

The reverse to the map described is easy to see.

\section{$5 \quad$ Extensions and open questions}

There are a number of questions that arise from this work. For example, our proofs of the formulas for $A_{s, k n+j}^{(k)}, B_{s, k n+j}^{(k)}, B_{0, s, k n+j}^{(k)}$, and $B_{1, s, k n+j}^{(k)}$ all arise by iterating simple recursion. It would be interesting to find more direct combinatorial proofs of these facts.

For example, we know that $A_{s, 2 n}^{(2)}=(n !)^{2}\left(\begin{array}{l}n \\ s\end{array}\right)^{2}$. We have direct combinatorial proofs in the case of $s=0$ and $s=n$. It is not difficult to extend those proofs to also prove the case $s=1$, but we do not have a simple combinatorial proof for general $s$.

Problem 1. A more general problem is to study pattern matching in permutations where one takes into account the equivalence classes of the element $\bmod k$ for some $k \geq 2$. That is, given any sequence $\sigma=\sigma_{1} \cdots \sigma_{n}$ of distinct integers, we let $\operatorname{red}(\sigma)$ be the permutation that results by replacing the $i$-th largest integer that appears in the sequence $\sigma$ by $i$. For example, if $\sigma=2754$, then $\operatorname{red}(\sigma)=1432$. Given a permutation $\tau$ in the symmetric group $S_{j}$, we define a permutation $\sigma=\sigma_{1} \cdots \sigma_{n} \in S_{n}$ to have a $\tau$-match at place $i$ provided $\operatorname{red}\left(\sigma_{i} \cdots \sigma_{i+j-1}\right)=\tau$. (In the literature, $\tau$-match is also called an occurrence of the consecutive pattern $\tau$.) Let $\tau$-mch $(\sigma)$ be the number of $\tau$-matches in the permutation $\sigma$. To prevent confusion, we note that a permutation not having a $\tau$-match is different than a permutation being $\tau$-avoiding. A permutation is called $\tau$-avoiding if there are no indices $i_{1}<\cdots<i_{j}$ such that $\operatorname{red}\left[\sigma_{i_{1}} \cdots \sigma_{i_{j}}\right]=\tau$. For example, if $\tau=2143$, then the permutation 321465 does not have a $\tau$-match but it does not avoid $\tau$ since $\operatorname{red}\left[\begin{array}{llll}2 & 1 & 6 & 5\end{array}\right]=\tau$.

In the case where $|\tau|=2$, then $\tau$-mch $(\sigma)$ reduces to familiar permutation statistics. That is, if $\sigma=\sigma_{1} \cdots \sigma_{n} \in S_{n}$, let $\operatorname{Des}(\sigma)=\left\{i: \sigma_{i}>\sigma_{i+1}\right\}$ and $\operatorname{Rise}(\sigma)=\left\{i: \sigma_{i}<\sigma_{i+1}\right\}$. Then it is easy to see that (2 1)-match $(\sigma)=\operatorname{des}(\sigma)=|\operatorname{Des}(\sigma)|$ and (1 2)-match $(\sigma)=$ 
$\operatorname{rise}(\sigma)=|\operatorname{Rise}(\sigma)|$.

We can consider a more refined pattern matching condition where we take into account conditions involving equivalence mod $k$ for some integer $k \geq 2$. That is, suppose we fix $k \geq 2$ and we are given some sequence of distinct integers $\tau=\tau_{1} \cdots \tau_{j}$. Then we say that a permutation $\sigma=\sigma_{1} \cdots \sigma_{n} \in S_{n}$ has a $\tau$-k-equivalence match at place $i$ provided $\operatorname{red}\left(\sigma_{i} \cdots \sigma_{i+j-1}\right)=\operatorname{red}(\tau)$ and for all $s \in\{0, \ldots, j-1\}, \sigma_{i+s}=\tau_{1+s} \bmod k$. For example, if $\tau=12$ and $\sigma=51743682$, then $\sigma$ has $\tau$-matches starting at positions 2, 5, and 6. However, if $k=2$, then only the $\tau$-match starting at position 5 is a $\tau$-2-equivalence match. Let $\tau$-k-emch $(\sigma)$ be the number of $\tau$-k-equivalence matches in the permutation $\sigma$.

More generally, if $\Upsilon$ is a set of sequences of distinct integers of length $j$, then we say that a permutation $\sigma=\sigma_{1} \cdots \sigma_{n} \in S_{n}$ has a $\Upsilon$ - $k$-equivalence match at place $i$ provided there is a $\tau \in \Upsilon$ such that $\operatorname{red}\left(\sigma_{i} \cdots \sigma_{i+j-1}\right)=\operatorname{red}(\tau)$ and for all $s \in\{0, \ldots, j-1\}, \sigma_{i+s}=\tau_{1+s}$ mod $k$. Let $\Upsilon$-k-emch $(\sigma)$ be the number of $\Upsilon$ - $k$-equivalence matches in the permutation $\sigma$ and $\Upsilon$-k-enlap $(\sigma)$ be the maximum number of non-overlapping $\Upsilon$ - $k$-equivalence matches in $\sigma$.

One can then study the following polynomials

$$
\begin{aligned}
T_{\tau, k, n}(x) & =\sum_{\sigma \in S_{n}} x^{\tau-k-e m c h(\sigma)}=\sum_{s=0}^{n} T_{\tau, k, n}^{s} x^{s} \text { and } \\
U_{\Upsilon, k, n}(x) & =\sum_{\sigma \in S_{n}} x^{\Upsilon-k-e m c h(\sigma)}=\sum_{s=0}^{n} U_{\Upsilon, k, n}^{s} x^{s} .
\end{aligned}
$$

Clearly our polynomials $A_{n}^{(k)}(x)$ and $B_{n}^{(k)}(x)$ are special cases of $U_{\Upsilon, k, n}(x)$ where all the patterns have length 2. Papers [7] and [8] have started the study of these more general types of polynomials in the case where all the patterns have length 2 .

Problem 2. There is another way to extend the results of this paper. That is, suppose that $X, Y$, and $Z$ are subsets of the natural numbers $N$. Then for any permutation $\sigma=\sigma_{1} \cdots \sigma_{n} \in S_{n}$, we can define

$$
\begin{aligned}
\operatorname{Des}_{X, Y, Z} & =\left\{i: \sigma_{i}>\sigma_{i+1}, \sigma_{i} \in X, \sigma_{i+1} \in Y, \& \sigma_{i}-\sigma_{i+1} \in Z\right\} \text { and } \\
\operatorname{des}_{X, Y, Z} & =\left|D e s_{X, Y, Z}\right| .
\end{aligned}
$$

One can then study the polynomials

$$
P_{n}^{X, Y, Z}(x)=\sum_{\sigma \in S_{n}} x^{\operatorname{des}_{X, Y, Z}}=\sum_{s \geq 0} P_{n, s}^{X, Y, Z} x^{s} .
$$

In [5], John Hall and the second author have shown that one can obtain formulas which are similar to the ones in this paper for the coefficients $P_{n, s}^{X, Y, Z}$ in the special case where $Z=N$. Moreover, they can give direct combinatorial proofs of these formulas so that they can give alternative proofs of some of the results in this paper. More generally, it is not difficult to see that the polynomials $P_{n}^{X, Y, Z}(x)$ are related to some special cases of hit 
polynomials for certain boards contained in the $n \times n$ rook board. Thus one can also use results on general formulas for hit polynomials such as those in [3] to study some of these polynomials. These results will be subject of future work by the authors, John Hall, and Jeff Liese.

\section{References}

[1] L. Comtet: Advanced Combinatorics, D. Reidel Publishing Co., Dordrecht, 1974.

[2] G. Gasper: Summation formulas for basic hypergeometric series, Siam. J. Math. Anal., 12 (1981), 196-200.

[3] James Haglund: Rook theory and hypergeometric Series, Advances in Applied Math., 17 (1996), 408-459.

[4] James Haglund: private communication.

[5] John Hall and Jeffrey B. Remmel: Counting descent pairs with prescribed tops and bottoms, in preparation.

[6] Sergey Kitaev and Jeffrey B. Remmel: Classifying descents according to parity, Annals of Combinatorics, to appear.

[7] Jeffrey Liese: Classifying ascents and descents with specified equivalences mod k, Proceedings of 18-th International Conference on Formal Power Series and Algebra Combinatorics, San Diego, CA (2006).

[8] Jeffrey Liese and Jeffrey B. Remmel: Pattern matching in permutaions relative to equivalence $\bmod k$, in preparation.

[9] P. A. MacMahon: Combinatory Analysis, Vol. 1 and 2, Cambridge Univ. Press, Cambridge, 1915 (reprinted by Chelsea, New York, 1955).

[10] M. Petkovsek, H. S. Wilf and D. Zeilberger: $A=B$. Wellesley, MA: A. K. Peters. 